\title{
Behavior of Electron-Spin Echoes and Photon Echoes in High Fields*
}

\author{
D. Grischkowsky† and S. R. Hartmann + \\ Columbia Radiation Laboratory, Department of Physics, Columbia University, New York, New York 10027 \\ (Received 15 May 1969; revised manuscript received 8 December 1969)
}

\begin{abstract}
The amplitude of the electron-spin or photon echo from an ensemble of isolated atoms (echo atoms) interacting with magnetic-nuclear neighbors is calculated as a function of the magnitude and the orientation of an applied magnetic field $\overrightarrow{\mathrm{H}}$ and the separation between the two excitation pulses, in the limit that the magnitude of $\overrightarrow{\mathrm{H}}$ is large compared to the effective local fields at the echo-atom sites due to the nuclear neighbors. The general problem is reduced to the equivalent problem of two-level echo atoms interacting with nuclear neighbors. The latter problem is readily solved and applied to both spin and photon echoes. Experimentally observed spin-echo behavior in ruby is compared to calculated spin-echo behavior using no adjustable parameters, and is found to be in good agreement even when the magnetic field is tilted away from the optic axis thereby causing the echoes to disappear. Calculated photon-echo behavior, in which it is assumed that the interaction constants in the excited state are simply related to those in the ground state, shows the same general features as those that have been observed in experiment. Several results are presented to show the wide range of photon-echo behavior for different values of the interaction constants. A simple theory is presented which explains most observed echo behavior in a straightforward way.
\end{abstract}

\section{INTRODUCTION}

Several experiments have been reported in which electron-spin echoes in ionic solids have been studied as a function of excitation pulse separation. ${ }^{1-4}$ These experiments were characterized by a modulated echo envelope; the modulation was caused by the interaction between the isolated electron spins and the nearby nuclear magnetic neighbors. Using the density-matrix formalism, ${ }^{5,6}$ Rowan, Hahn, and Mims gave the first rigorous theoretical treatment of the echo envelope modulation, where they considered the electron spins to be magnetically coupled to the nuclear neighbors by the isotropic hyperfine coupling and the direct dipole-dipole and pseudodipole anisotropic hyperfine interactions. ${ }^{3}$ Their calculation is valid for arbitrary spins for the paramagnetic ion (electron spin) and the nuclear neighbors. Applying the theory to $\mathrm{Ce}^{3+}$ in $\mathrm{CaWO}_{4}$ and characterizing the interaction between the electron spin and the nuclear neighbors by only two adjustable parameters, they were able to predict and observe the amplitude, width, and position of the initial dip in the echo envelope as a function of the angle $\theta_{0}$ between the magnetic field and the crystal $C$ axis. Zhidomirov and Salikov have theoretically studied the spin-echo envelope for the case of free radicals where the hyperfine structure is resolved. ${ }^{7}$

Recent observations of spin echoes in ruby also showed a modulated echo envelope (Fig. 1), where a straightforward density-matrix calculation similar to that of Rowan, Hahn, and Mims (except for the inclusion of a nuclear electric quadrupole term which is treated by perturbation theory) led to the good agreement of theory and experiment. ${ }^{4}$ However, to study an arbitrary transition between the energy levels of the $\mathrm{Cr}^{3+}$ ion with the magnetic field applied in any direction, a more general Hamiltonian had to be considered in the echo calculation, and the theoretical treatment was reformulated to handle this more complicated case in a simple manner. Using the theory developed in this paper, it is possible to treat the $\mathrm{Cr}^{3+}$ ion

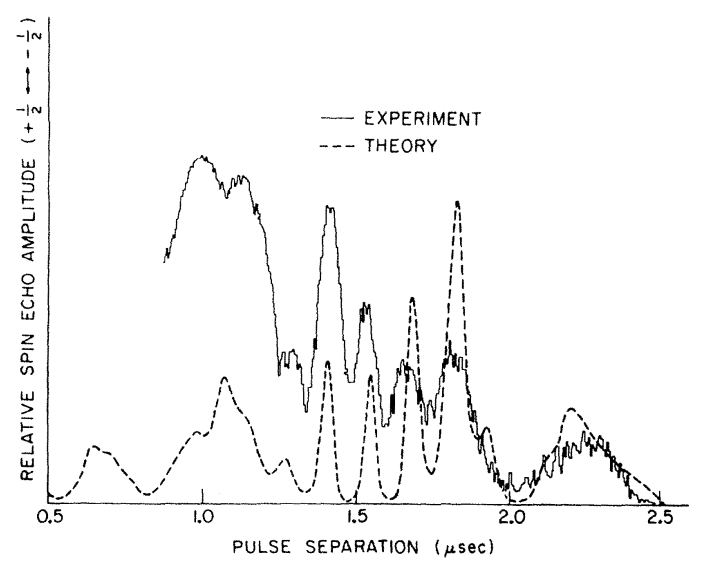

FIG. 1. Comparison of theory and experiment for the amplitude of the spin echoes in ruby versus the separation between the two excitation pulses; there were no adjustable parameters in the theory. The magnetic field of $3.3 \mathrm{kG}$ was applied along the optic axis, and the two excitation pulses had equal widths of approximately 60 nsec (Ref. 4). 
as a two-level system, and it is relatively easy to include crystalline field effects, electric quadrupole interactions, and more general energylevel structures in the calculation of the echo behavior. This generalized theory allows the echo envelope for the spin echoes in ruby to be calculated when the mixing of states of the $\mathrm{Cr}^{3+}$ ion caused by the crystal-field splitting becomes important $(\Theta \neq 0$, Figs. 4-6) and when the electric quadrupole term is no longer small compared to the other terms in the Hamiltonian for the nuclear neighbor (Fig. 4). No adjustable parameters are incorporated into the ruby spin-echo problem, since the ENDOR work of Laurance, McIrvine, and Lambe ${ }^{8}$ provides all the relevant interaction parameters. The theory is also readily applied to the calculation of the photon-echo behavior in ruby: ${ }^{9-12}$ The application is presently limited because of our lack of knowledge of the interaction Hamiltonian when the $\mathrm{Cr}^{3+}$ ions in $\mathrm{Al}_{2} \mathrm{O}_{3}$ are in the $\bar{E}\left({ }^{2} E\right)$ excited state; however, reasonable agreement with experiment is obtained when one assumes comparable interactions in the ground and excited states.

In order to provide insight into the echo-modulation process, a simple model is developed. The essence of this model is that the precessing, but otherwise randomly oriented, nuclear neighbors of the isolated echo atoms provide at the echoatom sites a quasiperiodic oscillating environment which modulates the energy separation between the two energy levels (echo states) in which the echo is forming and thereby leads to a modulation of the echo envelope. ${ }^{4}$

\section{GENERAL THEORY}

A quantum-mechanical calculation of the echo behavior, caused by the interaction between the echo atoms and the magnetic nuclear neighbors, is readily performed when static magnetic fields are applied which are large compared to the local magnetic fields at the atomic sites due to the neighbors and when nuclear spin-spin interactions are neglected. The calculation is greatly facilitated by describing the echo atom within the framework of the two levels (echo states) connected by the resonant radiation used to excite the echoes, while taking into account the interaction between the echo atom and the neighboring magnetic nuclei.

The Hamiltonian for a single echo atom in the host crystal, the nuclear neighbors, and the interaction between the echo atom and the neighbors is written

$$
\mathcal{H C}=\mathfrak{H}^{0}+\mathcal{H}_{R}^{0}+H^{n}+\mathcal{H}^{I},
$$

where $\mathfrak{F C}^{0}$ describes the interaction between the echo atom and the static environment, and $H^{n}$ is the corresponding term for the neighboring nuclei. $\mathcal{H}_{R}^{0}$ gives the time-dependent interaction between the atom and the oscillating radiation field. $\mathcal{F C}^{I}$ is the term responsible for the modulation of the echo envelope and describes the interaction between the echo atom and the nuclear neighbors.

The energy separation between the two echo states 1 and 2 of the echo atom is $\hbar \omega_{0}$, where level 1 has the higher energy. Because we apply intense resonant radiation of angular frequency $\omega_{0}$, only states 1 and 2 are strongly coupled together, and the influence of the other energy states of the echo atom is considered to be negligible. In many cases, for both electron spin and photon echoes, the interaction between the echo atom and the nuclear neighbors can cause transitions from echo states 1 and 2 to other states of the echo atom and thereby invalidate the two-level approximation. However, the effectiveness of this interaction in causing transitions is strongly field dependent, and if the applied field $\vec{H}$ is large enough compared to the local field at the echo atom site, these transitions do not occur, ${ }^{11}$ and the two-level approximation is valid. The following calculations assume approximation is correct. Consequently, the echo calculation can be made within the framework of the two echo states by replacing any operator $G$ by the reduced operator:

$$
\begin{aligned}
G= & \frac{1}{2}\left\{\left(G_{11}+G_{22}\right) \sigma_{0}+\left(G_{11}-G_{22}\right) \sigma_{3}\right. \\
& \left.+\left(G_{12}+G_{21}\right) \sigma_{1}+i\left(G_{12}-G_{21}\right) \sigma_{2}\right\}
\end{aligned}
$$

for $G_{\alpha \beta}=\langle\alpha|G| \beta\rangle$.

$|1\rangle$ and $|2\rangle$ are the eigenfunctions of $\mathcal{F}^{0}$ for the echo states 1 and 2, and the $\sigma$ 's are the Pauli matrices. By redefining zero energy for the system of an echo atom and the nuclear neighbors, we obtain the reduced Hamiltonian

$$
\begin{aligned}
\mathcal{H}= & =\frac{1}{2}\left\{\left(2 H^{n}+H_{11}^{I}+H_{22}^{I}\right) \sigma_{0}+\left(\hbar \omega_{0}+H_{11}^{I}-H_{22}^{I}\right) \sigma_{3}\right. \\
& \left.+\left(H_{R_{12}}^{0}+H_{R_{21}}^{0}\right) \sigma_{1}+i\left(H_{R_{12}}^{0}-H_{R_{21}}^{0}\right) \sigma_{2}\right\} . \text {. }
\end{aligned}
$$

The terms $H_{12}^{I}$ and $H_{21}^{I}$ are set equal to zero, since we consider only the situation where the frequency $\omega_{0}$, corresponding to the energy separation between the echo states, is very high compared to the precession frequencies of the neighboring nuclei, and the effects of these terms are averaged out. ${ }^{13} H_{R_{11}}^{0}$ and $H_{R_{22}}^{0}$ are neglected because they do not couple the two echo states.

The echo amplitude is proportional to the ensemble average of the time-dependent part of the expectation value of the dipole moment between the states 1 and 2 after the system has been excited by the two resonant pulses. Let $\overrightarrow{\mathrm{P}}$ designate the vector operator for the dipole moment of the echo atom and $\rho$ designate the density matrix for the 
system; the ensemble average of the expectation value of $\overrightarrow{\mathrm{P}}$ is then given by

$$
\langle\overrightarrow{\mathrm{P}}(t)\rangle=\operatorname{Tr} \overrightarrow{\mathbf{P}} \rho(t) \quad \text {. }
$$

For completeness, we review the essential points of the density-matrix theory which are applicable to our presentation. ${ }^{5,6}$ The density matrix $\rho$ satisfies the equation

$$
\frac{d \rho}{d t}=\frac{i}{\hbar}[\rho, \mathfrak{H}]
$$

When $\mathcal{H C}$ is time independent, Eq. (2.5) has the simple solution

$$
\begin{aligned}
\rho(t)= & \exp \left(\frac{-i}{\hbar}\left(t-t_{0}\right) \mathcal{H}\right) \\
& \times \rho\left(t_{0}\right) \exp \left(\frac{+i}{\hbar}\left(t-t_{0}\right) \mathfrak{H C}\right) .
\end{aligned}
$$

We apply two short intense resonant pulses of radiation to the system at $t=0$ and at $t=\tau$ and assume that during the application of the pulses $H^{n}$ and $\mathfrak{F C}^{I}$ can be neglected. Note that when the pulses are turned off, $\mathcal{H}_{R}^{0}$ disappears, and the Hamiltonian $\mathcal{H}$ is time independent. Thus, the density matrix at time $t(\tau<t)$ is

$$
\rho(t)=e^{-i(t-\tau) \nVdash / \hbar} R_{2} e^{-i \tau \pi / \hbar} R_{1} \rho^{0} \text { (H.c.) , }
$$

where $R_{1}$ and $R_{2}$ give the effect of the excitation pulses, and H.c. is the Hermitian conjugate of the product of operators acting on the initial density matrix $\rho^{0}$ from the left. $R_{1}$ and $R_{2}$ have the form of simple rotation operators, and when the twopulse sequence is the ideal $90^{\circ}-180^{\circ}$ series, they are given by

$$
R_{1}=\exp \left(-\frac{1}{4} i \pi \sigma_{2}\right) \quad \text { and } \quad R_{2}=\exp \left(-\frac{1}{2} i \pi \sigma_{2}\right) \text {. }
$$

However, the echo behavior due to the interaction between the echo atoms and the nuclear neighbors is the same for any other two-pulse sequence $\varphi_{1}$ and $\varphi_{2}\left(\varphi_{1}\right.$ and $\varphi_{2}$ are the angles by which $\overrightarrow{\mathrm{P}}$ is rotated about the fields of the first and second excitation pulses, respectively), but the strength of the echo is reduced by the factor $\sin \varphi_{1} \sin ^{2}\left(\frac{1}{2} \varphi_{2}\right)$. The Hamiltonian $\mathcal{H}$ in Eq. (2.7) does not contain $\mathcal{H}_{R}^{0}$, the radiation field interaction term, and we find it convenient to introduce the operator

$$
\mathcal{H}_{ \pm}=\frac{1}{2}\left(2 H^{n}+H_{11}^{I}+H_{22}^{I}\right) \sigma_{0} \pm \frac{1}{2}\left(H_{11}^{I}-H_{22}^{I}\right) \sigma_{3} ;
$$

the \pm notation will be quite useful. The expression for $\rho(t)$ now becomes

$$
\rho(t)=\left\{\exp \left[-\frac{1}{2} i(t-2 \tau) \omega_{0} \sigma_{3}\right]\right\}\left\{\exp \left[-i(t-\tau) \mathcal{H}_{+} / \hbar\right]\right\} R_{2}\left\{\exp \left[-i \tau \mathcal{H}_{+} / \hbar\right]\right\} R_{1} \rho^{0} \text { (H. c.) . }
$$

The operator $\rho^{0}$ for the initial density matrix of the system before any pulses are applied can be written

$$
\rho^{0}=\left[\frac{1}{2} \sigma_{0}-\frac{1}{2} \sigma_{3} \tanh \left(\hbar \omega_{0} / 2 k T\right)\right] \Pi_{j}\left(2 I_{j}+1\right)^{-1},
$$

where the ensemble of echo atoms is assumed to have temperature $T$ and the nuclear neighbors are treated by the high-temperature approximation. The index $j$ refers to the $j$ th neighbor, and $I_{j}$ is the nuclear spin quantum number. Because the echo amplitude is proportional to the time-dependent part of $\langle\overrightarrow{\mathbf{P}}(t)\rangle$, only the time-dependent part of the density matrix is important for the echo calculation, and the first term of the initial density matrix, which allows all of the time-development operators to collapse, can be neglected. Recalling that $\exp \left(-i \varphi \sigma_{2}\right)$ describes a rotation of $2 \varphi$ rad and using the identity

$$
\sigma_{1}=-i \exp \left(+\frac{1}{2} i \pi \sigma_{1}\right)
$$

we can change Eq. (2.10) to

$$
\begin{aligned}
\rho(t)= & \frac{1}{2} \tanh \frac{1}{2}\left(\hbar \omega_{0} / k T\right) \Pi_{j}\left(2 I_{j}+1\right)^{-1} \sigma_{1} \exp \left[i(t-2 \tau) \omega_{0} \sigma_{3}\right] \exp \left[-i(t-\tau) \mathcal{H}_{-} / \hbar\right] \\
& \times \exp \left[-i \tau \mathcal{H}_{+} / \hbar\right] \exp \left[+i \tau \mathcal{H}_{-} / \hbar\right] \exp \left[+i(t-\tau) \mathcal{H}_{+} / \hbar\right] .
\end{aligned}
$$

We now define new operators $H_{1}$ and $H_{2}$, so that $\mathcal{H}_{ \pm}$can be written more symmetrically as

$$
\mathfrak{H}_{ \pm}=\frac{1}{2}\left(H_{1}+H_{2}\right) \sigma_{0} \pm \frac{1}{2}\left(H_{1}-H_{2}\right) \sigma_{3} \text {. }
$$

Because we neglect interactions among the nuclear neighbors, the Hermitian operators $H_{1}$ and $H_{2}$ are decomposable into two series of Hermitian operators; each operator in a series acts on a single nuclear neighbor.

$$
H_{1}=H^{n}+H_{11}^{I}=\sum_{j} h_{1}^{j} \quad \text { and } \quad H_{2}=H^{n}+H_{22}^{I}=\sum_{j} h_{2}^{j} \quad \text {. }
$$

The commutation relations $\left[h_{1}^{i}, h_{1}^{j}\right]=\left[h_{1}^{i}, h_{2}^{j}\right]=\left[h_{2}^{i}, h_{2}^{j}\right]=0$

are satisfied by these operators for $i \neq j$. The indices $i$ and $j$ refer to the $i$ th and $j$ th nuclear neighbors, 
respectively. Using Eq. (2.14), we can expand Eq. (2.13) as

$$
\begin{aligned}
\rho(t)= & \frac{1}{4} \tanh \left(\hbar \omega_{0} / 2 k T\right)\left\{\left(\left(C+C^{\dagger}\right) \cos \left[(t-2 \tau) \omega_{0}\right]+i\left(C-C^{\dagger}\right) \sin \left[(t-2 \tau) \omega_{0}\right]\right) \sigma_{1}\right. \\
& \left.+\left(-i\left(C-C^{\dagger}\right) \cos \left[(t-2 \tau) \omega_{0}\right]+\left(C+C^{\dagger}\right) \sin \left[(t-2 \tau) \omega_{0}\right]\right) \sigma_{2}\right\}, \\
\text { with } C & =\Pi_{j}\left(2 I_{j}+1\right)^{-1} \exp \left[-i(t-\tau) H_{2} / \hbar\right] \exp \left[-i \tau H_{1} / \hbar\right] \exp \left[+i \tau H_{2} / \hbar\right] \exp \left[+i(t-\tau) H_{1} / \hbar\right] .
\end{aligned}
$$

Referring to Eq. (2.15), we note that $C=\Pi_{j} C_{j}$,

with $\quad C_{j}=\left(2 I_{j}+1\right)^{-1} \exp \left[-i(t-\tau) h_{2}^{j} / \hbar\right] \exp \left[-i \tau h_{1}^{j} / \hbar\right] \exp \left[i \tau h_{2}^{j} / \hbar\right] \exp \left[i(t-\tau) h_{1}^{j} / \hbar\right]$.

$\langle\overrightarrow{\mathrm{P}}(t)\rangle$ given by Eq. (2.4) can be written

$$
\begin{aligned}
\langle\overrightarrow{\mathrm{P}}(t)\rangle & =\frac{1}{4} \tanh \left(\hbar \omega_{0} / 2 k T\right) \Pi_{j} \operatorname{Tr}\left\{\left[\overrightarrow{\mathrm{P}}_{12}+\overrightarrow{\mathrm{P}}_{21}\right]\left[\left(C_{j}+C_{j}^{\dagger}\right) \cos (t-2 \tau) \omega_{0}+i\left(C_{j}-C_{j}^{\dagger}\right) \sin (t-2 \tau) \omega_{0}\right]\right. \\
& \left.+\left[\overrightarrow{\mathrm{P}}_{12}-\overrightarrow{\mathrm{P}}_{21}\right]\left[\left(C_{j}-C_{j}^{\dagger}\right) \cos (t-2 \tau) \omega_{0}+i\left(C_{j}+C_{j}^{\dagger}\right) \sin (t-2 \tau) \omega_{0}\right]\right\},
\end{aligned}
$$

which reduces to the relatively simple equation

$$
\begin{aligned}
\langle\overrightarrow{\mathrm{P}}(t)\rangle & =\tanh \left(\hbar \omega_{0} / 2 k T\right) \\
& \times \operatorname{Re} \exp \left[i(t-2 \tau) \omega_{0}\right] \overrightarrow{\mathrm{P}}_{12} \Pi_{j} \operatorname{Tr} C_{j} .
\end{aligned}
$$

Equation $(2.22)^{13 a}$ is the central result of this section. The magnitude of the oscillating dipole moment at $t=2 \tau$ (and hence the echo amplitude) is expressed quite simply by Eq. (2.22) in terms of $\overrightarrow{\mathrm{P}}_{12}$, the matrix element of the dipole moment between the two echo states, and $\Pi_{j} \operatorname{Tr} C_{j}$, the product of the traces of $C_{j}$ for each nuclear neighbor. In simple cases, $\operatorname{Tr} C_{j}$ can be evaluated exactly, and for the more difficult cases $\operatorname{Tr} C_{j}$ can be evaluated numerically. The echo behavior due to the interaction between the echo atom and the nuclear neighbors is completely determined by $\Pi_{j} \operatorname{Tr} C_{j}$, and in the following sections $\Pi_{j} \operatorname{Tr} C_{j}$ is evaluated for the two particular cases of electron-spin and photon echoes in ruby.

The echo occurs at $t \approx 2 \tau$, and for evaluation of the echo signal the slowly varying term $C_{j}$ is set equal to

$$
\begin{aligned}
C_{j}= & \left(2 I_{j}+1\right)^{-1} \exp \left[-i \tau h_{2}^{j} / \hbar\right] \exp \left[-i \tau h_{1}^{j} / \hbar\right] \\
& \times \exp \left[+i \tau h_{2}^{j} / \hbar\right] \exp \left[+i \tau h_{1}^{j} / \hbar\right] .
\end{aligned}
$$

Because $C_{j}$ is a product of functions of the operators $h_{1}^{j}$ and $h_{2}^{j}$, it is necessary that we be able to diagonalize these operators. Accordingly, we define ${ }^{j} U_{1}$ and ${ }^{j} U_{2}$ so that

$$
{ }^{j} U_{1} h_{1}^{j j} U_{1}^{\dagger}=h_{1 D}^{j} \text { and }{ }^{j} U_{2} h_{2}^{j j} U_{2}^{\dagger}=h_{2 D}^{j} \text {, }
$$

where $h_{1 D}^{j}$ and $h_{2 D}^{j}$ are diagonal matrices. All of the results are being derived for the $j$ th neighbor; however, to keep the number of indices to a minimum, the $j$ subscripts and superscripts will be dropped. The trace of $C_{j}$ can be put in the form

$\operatorname{Tr} C_{j}=\left(2 I_{j}+1\right)^{-1} \operatorname{Tr} U_{2}^{\dagger} U_{2} \exp \left[-i \tau h_{2} / \hbar\right]$

$$
\begin{aligned}
& \times U_{2}^{\dagger} U_{2} U_{1}^{\dagger} U_{1} \exp \left[-i \tau h_{1} / \hbar\right] U_{1}^{\dagger} U_{1} U_{2}^{\dagger} U_{2} \\
& \times \exp \left[+i \tau h_{2} / \hbar\right] U_{2}^{\dagger} U_{2} U_{1}^{\dagger} U_{1} \exp \left[+i \tau h_{1} / \hbar\right] U_{1}^{\dagger} U_{1},
\end{aligned}
$$

which is equivalent to

$$
\begin{aligned}
\operatorname{Tr} C_{j}= & \left(2 I_{j}+1\right)^{-1} \operatorname{Tr} \exp \left[-i \tau h_{2 D} / \hbar\right] W \\
& \times \exp \left[-i \tau h_{1 D} / \hbar\right] W^{\dagger} \exp \left[+i \tau h_{2 D} / \hbar\right] W \\
& \times \exp \left[i \tau h_{1 D} / \hbar\right] W^{\dagger},
\end{aligned}
$$

with $W=U_{2} U_{1}^{\dagger} . W$ is a time-independent unitary matrix; all of the time dependence is carried by the diagonal matrices. Equation (2.26) can be written in a form more suitable for direct evaluation as

$$
\begin{aligned}
& \operatorname{Tr}_{j}=\left(2 I_{j}+1\right)^{-1}\left(\sum _ { \alpha , \gamma , \lambda , \epsilon } ^ { 2 I + 1 } \left\{\cos \left[\tau\left(h_{2 D_{\alpha}}-h_{2 D_{\lambda}}\right) / \hbar\right]\right.\right. \\
& \times \cos \left[\tau\left(h_{1 D_{\gamma}}-h_{1 D_{\epsilon}}\right) / \hbar\right]-\sin \left[\tau\left(h_{2 D_{\alpha}}-h_{2 D_{\lambda}}\right) / \hbar\right] \\
& \left.\left.\times \sin \left[\gamma\left(h_{1 D_{\gamma}}-h_{1 D_{\epsilon}}\right) / \hbar\right]\right\} W_{\alpha \gamma} W_{\alpha \epsilon}^{*} W_{\lambda \gamma} W_{\lambda \epsilon}\right)_{j},
\end{aligned}
$$

with $\quad\left(h_{1 D}\right)_{\alpha \alpha}=h_{1 D_{\alpha}}$ and $\left(h_{2 D}\right)_{\beta \beta}=h_{2 D_{\beta}}$.

$h_{1 D_{\alpha}}$ or $h_{2 D_{\beta}}$ are the energy eigenvalues of the $j$ th nuclear neighbor with the echo atom in echo state 1 or 2 , respectively. $W_{\alpha \epsilon}^{*}$ is the complex conjugate of the matrix element $W_{\alpha \epsilon}$. The summation indices $\alpha, \gamma, \lambda$, and $\epsilon$ are independent and range from 1 to $2 I+1$, where $I$ is the nuclear spin quantum number. The $j$ subscript on the right indicates that all the quantities in the brackets must be evaluated for the $j$ th nuclear neighbor. The evaluation of $\operatorname{Tr} C_{j}$ yields the echo amplitude through Eq. (2.22).

It is interesting to note that $\operatorname{Tr} C_{j}$ is expressible as a summation of products of pairs of sines and cosines with each pair having one term oscillating at a precession frequency associated with the echo atom being in the ground state (echo state 2 ) and 
the other term oscillating at a precession frequency associated with the echo atom being in the excited state (echo state 1).

\section{SPIN ECHOES IN RUBY}

\section{A. Theory}

Using the general theory of Sec. II as our basic theoretical framework, we will calculate the spinecho behavior in ruby due to the interactions between the paramagnetic $\mathrm{Cr}^{3+}$ ions and the $\mathrm{Al} \mathrm{nu-}$ clear neighbors. The electron-spin echoes are associated with the various allowed transitions between the different spin states (shown in Fig. 2) of the $\mathrm{Cr}^{3+}$ ion. The Hamiltonian describing a single $\mathrm{Cr}^{3+}$ impurity ion in $\mathrm{Al}_{2} \mathrm{O}_{3}$ (ruby) and the $\mathrm{Al}$ neighbors is considered to have the form ${ }^{8}$

$$
\begin{aligned}
\mathcal{H}= & g \mu_{\beta} \overrightarrow{\mathrm{H}} \cdot \overrightarrow{\mathrm{S}}+D\left[\left(S_{z}\right)^{2}-\frac{1}{3} S(S+1)\right] \\
& +\sum_{j}\left\{-\hbar \gamma \overrightarrow{\mathrm{H}} \cdot \overrightarrow{\mathrm{I}}_{j}+Q^{j}\left[\left(I_{z j}\right)^{2}-\frac{1}{3} I(I+1)\right]\right\} \\
& +\overrightarrow{\mathrm{S}} \cdot \sum_{j}\left\{A_{j} \overrightarrow{\mathrm{I}}_{j}+B_{j}\left[3 \overrightarrow{\mathrm{r}}_{j}\left(\overrightarrow{\mathrm{I}}_{j} \cdot \overrightarrow{\mathrm{r}}_{j}\right) / \gamma_{j}^{5}-\overrightarrow{\mathrm{I}}_{j} / \gamma_{j}^{3}\right]\right\} .
\end{aligned}
$$

For Eq. (3.1), the $z$ axis is parallel to the axis of trigonal symmetry (the optic axis) for the crystal, and the $\mathrm{Cr}^{3+}$ ion is located at the origin of coordinates. The $g$ value for $\mathrm{Cr}^{3+}$ is considered to be isotropic with $g=1.984 ; \mu_{\beta}$ is the Bohr magneton. The symbol $D$ is the measure of the crystal-field splitting, and $2 \mathrm{D} / \hbar=-11.493 \mathrm{GHz}$. The summation is taken over the 13 nearest $\mathrm{Al}$ neighbors. $\overrightarrow{\mathrm{S}}$ and $\overrightarrow{\mathrm{I}}_{j}$ are the spin operators for the $\mathrm{Cr}^{3+}$ ion and the $j$ th $\mathrm{Al}$ nucleus, respectively, where the $\mathrm{Cr}^{3+}$ ion has spin $\frac{3}{2}$ and the $\mathrm{Al}$ neighbors have nuclear spin $\frac{5}{2}$. $\overrightarrow{\mathrm{H}}$ is the applied magnetic field; $\gamma / 2 \pi$ for the $\mathrm{Al}$ nucleus is $11.094 \mathrm{MHz}$ for a field of $10 \mathrm{kG}$. In Table I, we give the values of $A_{j}, B_{j}, Q^{j}$, and $\overrightarrow{\mathrm{r}}_{j}$ used in our computer calculations. The constants $A_{j}, B_{j}$, and $Q^{j}$ for the 13 nearest neighbors have been determined from earlier ENDOR work (Table

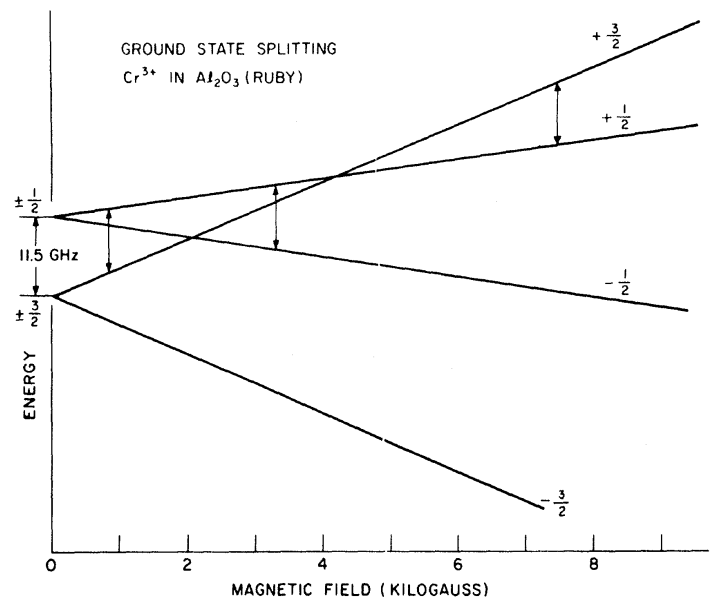

FIG. 2. Splitting of the ${ }^{4} A_{2}$ ground state of the $\mathrm{Cr}^{3+}$ ion versus the strength of the magnetic field applied along the optic axis. The three arrows indicate the allowed transitions at $9.25 \mathrm{GHz}$.

4 of Laurance et $a l .^{8}$ ) by the relations

$$
\begin{aligned}
& A_{j}=h\left[\left(A+B_{z}\right)_{\text {expt }}-\left(B_{t \text { expt }} / B_{t \text { point }}\right) B_{z \text { point }}\right] \times 10^{6}, \\
& B_{j}=\left(B_{t \text { expt }} / B_{t \text { point }}\right) \mu_{\beta} g \hbar \gamma \\
& Q^{j}=h Q^{\prime} \times 10^{6},
\end{aligned}
$$

where the subscript expt refers to their experimental values given in $\mathrm{MHz}$ and the subscript point refers to their calculated result for a point dipole. The position vectors $\vec{r}_{j}$ with components $\left(x_{j}, y_{j}, z_{j}\right)$ of the nuclear neighbors with the $\mathrm{Cr}^{3+}$ ion at the origin of coordinates have been obtained from $\mathrm{Ta}$ ble 1 of Ref. 8. Laurance et al. designate the four sets of equivalent nuclear neighbors as sets $I, J, K$, and $L$ and the nearest neighbor by $G$. In our notation set $I$ corresponds to neighbors 1-3; set $J$ corresponds to neighbors 4-6; set $K$ corresponds to neighbors 7-9; set $L$ corresponds to

TABLE I. Parameters $A_{j}, B_{j}, Q^{j}$, and $\overrightarrow{\mathrm{r}}_{j}$ for the 13 nearest $\mathrm{Al}$ neighbors of the $\mathrm{Cr}^{3+}$ ion in ruby.

\begin{tabular}{ccccccc}
\hline \hline $\begin{array}{c}\text { Nuclear } \\
\text { neighbor }\end{array}$ & $\begin{array}{c}A_{j} \\
\left(10^{-20} \mathrm{erg}\right)\end{array}$ & $\begin{array}{c}B_{j} \\
\left(10^{-43} \mathrm{erg} \mathrm{cm}\right)\end{array}$ & $\begin{array}{c}Q^{j} \\
\left(10^{-20} \mathrm{erg}\right)\end{array}$ & $\begin{array}{c}x_{j} \\
\left(10^{-8} \mathrm{~cm}\right)\end{array}$ & $\begin{array}{c}y_{j} \\
\left(10^{-8} \mathrm{~cm}\right)\end{array}$ & $\begin{array}{c}z_{j} \\
\left(10^{-8} \mathrm{~cm}\right)\end{array}$ \\
\hline 1 & 2.04 & 1.08 & 0.093 & +1.380 & +2.380 & -0.560 \\
2 & 2.04 & 1.08 & 0.093 & +1.380 & -2.380 & -0.560 \\
3 & 2.04 & 1.08 & 0.093 & -2.751 & 0.0 & -0.560 \\
4 & 1.56 & 1.72 & 0.113 & +2.751 & 0.0 & -2.170 \\
5 & 1.56 & 1.72 & 0.113 & -1.380 & +2.380 & -2.170 \\
6 & 1.56 & 1.72 & 0.113 & -1.380 & -2.380 & -2.170 \\
7 & 1.46 & 1.36 & 0.096 & +2.751 & 0.0 & +1.600 \\
8 & 1.46 & 1.36 & 0.096 & -1.380 & +2.380 & +1.600 \\
9 & 1.46 & 1.36 & 0.096 & -1.380 & -2.380 & +1.600 \\
10 & 0.86 & 1.70 & 0.116 & +1.380 & +2.380 & +2.170 \\
11 & 0.86 & 1.70 & 0.116 & +1.380 & -2.380 & +2.170 \\
12 & 0.86 & 1.70 & 0.116 & -2.751 & 0.0 & +2.170 \\
13 & 0.45 & 1.35 & 0.175 & 0.0 & 0.0 & -2.730 \\
\hline
\end{tabular}


neighbors $10-12$, and $G$ corresponds to neighbor 13.

We diagonalize the spin Hamiltonian ${ }^{14}$ for the $\mathrm{Cr}^{3+}$ ion and obtain the four eigenstates. However, we are only concerned with the eigenfunctions des ignated as $|1\rangle$ and $|2\rangle$, which are involved in the spin-echo formation. Note that when the angle $\theta$ between the optic axis and $\overrightarrow{\mathrm{H}}$ is not zero, the states $|1\rangle$ and $|2\rangle$ are not eigenfunctions of $S_{z}$, and the vectors $\overrightarrow{\mathrm{S}}_{11}$ and $\overrightarrow{\mathrm{S}}_{22}$ are not parallel to $\overrightarrow{\mathrm{H}}$. Equation (2.4) gives the spin-echo amplitude with $\vec{P}$ the dipole moment operator given by

$$
\overrightarrow{\mathrm{P}}=-g \mu_{\beta} \overrightarrow{\mathrm{S}} \text {. }
$$

The evaluation of $\operatorname{Tr} C_{j}$ for spin echoes is accomplished by writing $h_{1,2}^{j}$ of Eq. (2.15) as

$$
\begin{aligned}
h_{1,2}^{j}-\hbar \gamma\left[\overrightarrow{\mathrm{H}}-\frac{A_{j} \overrightarrow{\mathrm{S}}_{11,22}}{\hbar \gamma}-\frac{B_{j}}{\hbar \gamma}\left(\frac{3 \overrightarrow{\mathrm{r}}_{j}\left(\overrightarrow{\mathrm{S}}_{11,22} \cdot \overrightarrow{\mathrm{r}}_{j}\right)}{r_{j}^{5}}\right.\right. \\
\left.\left.\quad-\frac{\overrightarrow{\mathrm{S}}_{11,22}}{r_{j}^{3}}\right)\right] \cdot \overrightarrow{\mathrm{I}}_{j}+Q_{j}\left\{I_{z j}^{2}-\frac{1}{3}[I(I+1)]\right\} 。
\end{aligned}
$$

Equation (3.4) and the following equations are actually two separate equations, one for $h_{1}^{j}$ and the other for $h_{2}^{j}$, according to whether one uses only the 1 or 2 indices, respectively. Equation (3.4) can be recast into a form which uses the concept of the effective magnetic fields ${ }^{8} \overrightarrow{\mathrm{H}}_{\epsilon j}^{1,2}$ at the $j$ th $\mathrm{Al}$ neighbor.

$$
\begin{aligned}
h_{1,2}= & -\hbar \gamma\left|\overrightarrow{\mathrm{H}}_{\epsilon}^{1,2}\right|\left(I_{x} \sin \eta_{1,2} \cos \chi_{1,2}+I_{y} \sin \eta_{1,2} \sin \chi_{1,2}\right. \\
& \left.+I_{z} \cos \eta_{1,2}\right)+Q\left[I_{z}^{2}-\frac{1}{3} I(I+1)\right] ;
\end{aligned}
$$

the $j$ indices are neglected for clarity. We write the operators $U_{1,2}$ of Eq. (2.24), which diagonalize $h_{1,2}$ as the products

$$
U_{1,2}=V_{1,2} T_{1,2},
$$

where $T_{1,2}$ are simple rotation operators such that

$$
\begin{aligned}
T_{1,2} h_{1,2} T_{1,2}^{\dagger}= & -\hbar \gamma\left|\overrightarrow{\mathrm{H}}_{\epsilon}^{1,2}\right|\left(I_{x} \sin \eta_{1,2}\right. \\
& \left.+I_{z} \cos \eta_{1,2}\right)+Q\left[I_{z}^{2}-\frac{1}{3} I(I+1)\right] \quad .(3.7)
\end{aligned}
$$

Equation (3.7) defines two real symmetric, $6 \times 6$ matrices which are easily diagonalized numerically to yield $h_{1 D, 2 D}$ and $V_{1,2}$. The $\operatorname{Tr} C_{j}$ is then obtained from Eq. (2.27).

\section{B. Experiment}

The spin-echo spectrometer is shown schematically in Fig。3. The sample is contained in the low $-Q$ balanced bimodal cavity, which is immersed in liquid helium, and the two microwave excitation pulses are generated by the magnetron mechanically tuned to the cavity frequency. During the excitation pulses, the receiver is protected by the three units in front of the mixer; the limiter, the isolator, and the Hewlett-Packard PIN modulator. After the

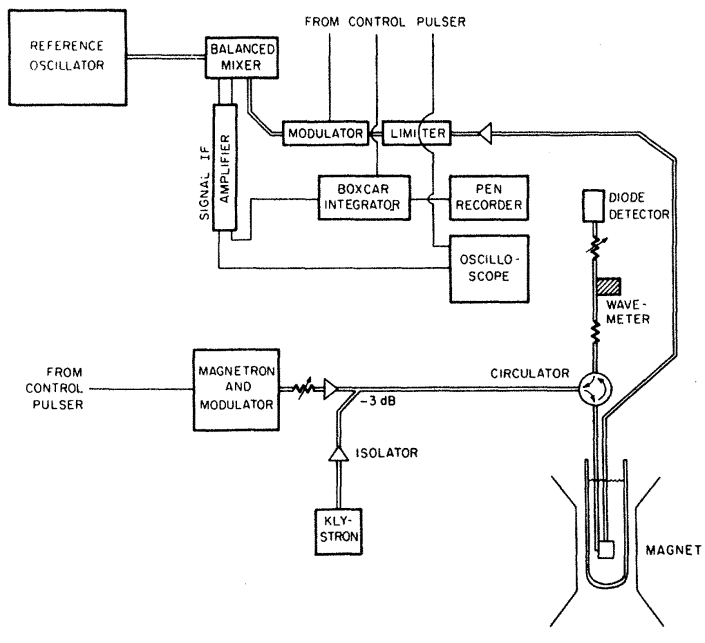

FIG. 3. Spin-echo spectrometer.

two pulses, the attenuation due to the PIN modulator is turned off, thereby effectively connecting the receiver to the output waveguide of the bimodal cavity. If an echo is formed, the oscillating magnetization of the sample spontaneously rephases and then dephases, and a microwave echo pulse at the cavity frequency is radiated by the sample. The echo pulse is mixed with the continuous reference signal with a frequency $30 \mathrm{MHz}$ above or below the cavity frequency, and an i.f. output pulse is emitted by the balanced mixer at the difference frequency. This $30-\mathrm{MHz}$ pulse is amplified by the signal i.f. amplifier and observed with the oscilloscope, or the amplified pulse is detected and put into the boxcar integrator, which is pulsed on during the echo pulse. The dc output of the boxcar is proportional to the amplitude of the detected echo pulse. The gating pulse to the boxcar is $0.5 \mu \mathrm{sec}$ wide and the i.f. amplifier has a bandpass of $2 \mathrm{MHz}$. The data taking is completely automated; the spacing between the two excitation pulses is slowly changed by a clock motor, and the magnitudes of the resulting echo pulses are recorded by a chart recorder connected to the boxcar output. The resulting record gives the echo amplitude versus the separation between the two excitation pulses.

Several initial attempts to observe electron-spin echoes in ruby at liquid-helium temperatures were unsuccessful. With reference to other related experiments, ${ }^{8,9}$ it was inferred that the magnetic field $\overrightarrow{\mathrm{H}}$ should be applied precisely along the optic axis of the crystal and that the concentration of the $\mathrm{Cr}^{3+}$ ion should be reduced. When the concentration of $\mathrm{Cr}_{2} \mathrm{O}_{3}$ was reduced from 0.05 to $0.005 \%$ by weight and $\overrightarrow{\mathrm{H}}$ was applied parallel to the optic axis of the crystal, echoes were observed. $\overrightarrow{\mathrm{H}}$ was aligned parallel to the optic axis by monitoring the 


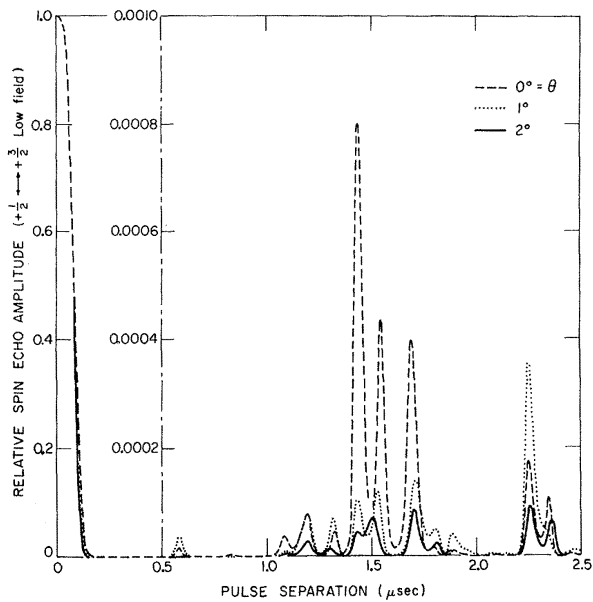

FIG. 4. Theoretical dependence for the $\left(+\frac{1}{2} \longleftrightarrow+\frac{3}{2}\right.$ low-field) transition at $9.25 \mathrm{GHz}$ shown in Fig. 2, of the amplitude of the spin echoes in ruby on the separation between the two excitation pulses as the angle $\theta$ between the optic axis and the magnetic field is increased. Note the scale changes at $0.5 \mu \mathrm{sec}$.

resonance signal associated with the "forbidden" $\left(+\frac{3}{2} \multimap-\frac{1}{2}\right)$ transition and by adjusting the orientation of $\overrightarrow{\mathrm{H}}$ for minimum signal. Because of the long spin-lattice relaxation time of the $\mathrm{Cr}^{3+}$ ion in ruby at liquid-helium temperatures, the repetition rate for echo production had to be of the order of one pulse series per sec.

\section{Results}

The theoretical dependence of the spin-echo

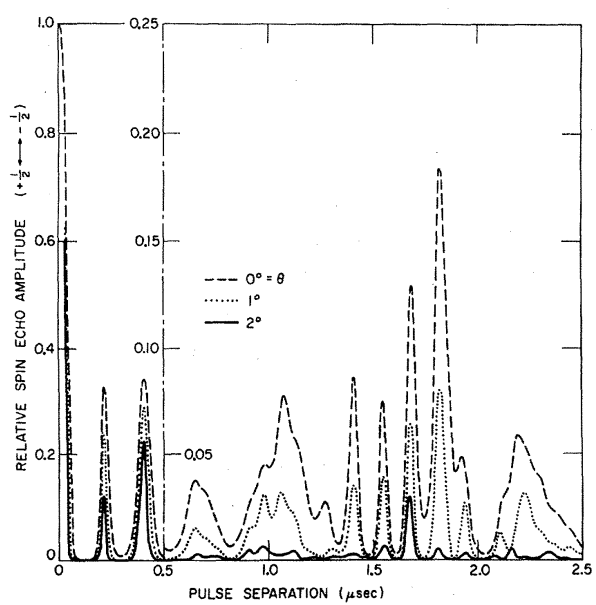

FIG. 5. Theoretical dependence, for the $\left(+\frac{1}{2}--\frac{1}{2}\right)$ transition at 9.25 GHz shown in Fig. 2, of the amplitude of the spin-echoes in ruby on the separation between the two excitation pulses as the angle $\theta$ between the optic axis and the magnetic field is increased. Note the scale changes at $0.5 \mu \mathrm{sec}$.

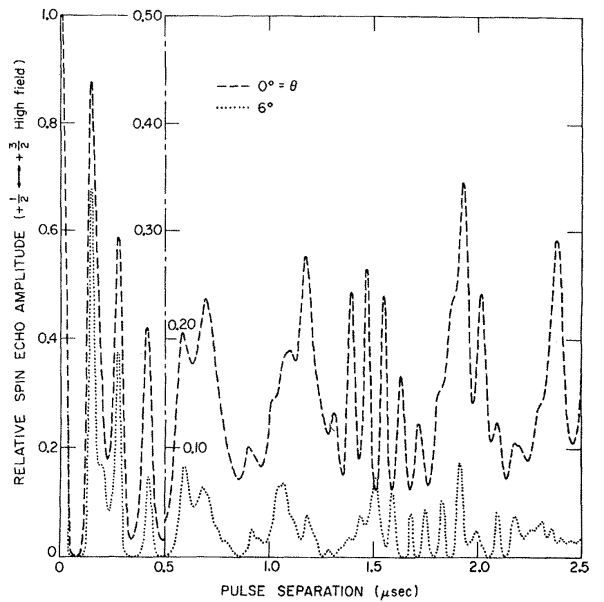

FIG. 6. Theoretical dependence, for the $\left(+\frac{1}{2} \longleftrightarrow+\frac{3}{2}\right.$ high-field) transition at 9.25 GHz shown in Fig. 2, of the amplitude of the spin echoes in ruby on the separation between the two excitation pulses as the angle $\theta$ between the optic axis and the magnetic field is increased. Note the scale changes at $0.5 \mu$ sec.

amplitude on the separation $\tau$ between the two excitation pulses and on the angle $\theta$ between the applied magnetic field $\overrightarrow{\mathrm{H}}$ and the optic axis of the ruby crystal is presented in Figs. 4-6 for the three allowed transitions at $9.25 \mathrm{GHz}$, which are indicated by the arrows in Fig. 2. Figures $4-6$ show the same general behavior: The amplitude of the echo signal is modulated as $\tau$ is changed, and the echo signal is reduced as $\theta$ is increased. Also, the average echo signal increases as $\overrightarrow{\mathrm{H}}$ is raised from the low-field transition to the high-field transition. Figure 7 shows the separate contributions $\left(\operatorname{Re} \operatorname{Tr} C_{j}\right)$ from each nuclear-neighbor type to the modulation pattern. With $\Theta=0$ there are three neighbors of each type, and the modulation is given by the product of the curves for each type raised to the third power. The result $\left(\operatorname{Re} \Pi_{j} \operatorname{Tr} C_{j}\right)$ is the bottom curve of Fig. 7, which is compared with experiment in Fig. 1. The theoretical curves have been normalized to unity for zero pulse separation and are proportional to the real part of $\Pi_{j} \operatorname{Tr} C_{j}$ [see Eqs. (2.22) and (2.27)], as the imaginary part is negligible for small $\Theta$. For the three transitions, the magnitude of $\overrightarrow{\mathrm{H}}$ was adjusted to maintain the energy separation of $9.25 \mathrm{GHz}$ between the two states involved in the echo formation as $\theta$ was changed. The echo amplitude shows a minor dependence on the angular orientation of the plane containing $\overrightarrow{\mathrm{H}}$ and the optic axis. However, with certain exceptions the major features of the theoretical curves are essentially unchanged as $\vec{H}$ is rotated about the optic axis with $\Theta$ kept constant. No adjustable parameters were involved in the 


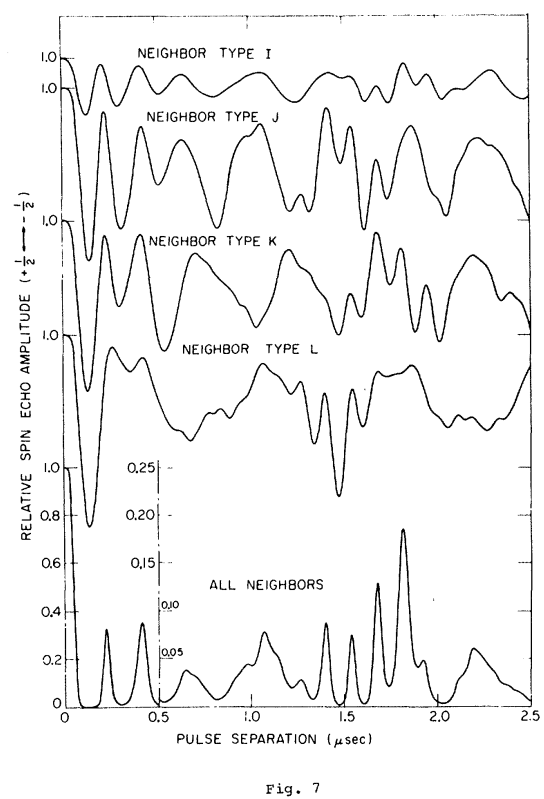

FIG. 7. Decomposition of the theoretical spin-echo envelope modulation for the $\left(+\frac{1}{2} \longleftrightarrow-\frac{1}{2}\right)$ transition at 9.25 GHz with $\theta=0$, which shows the modulation that would be observed if the $\mathrm{Cr}^{3+}$ ion interacted with a single Al nuclear neighbor of type I, $J, K$, or $L$, (Ref. 8) respectively. Since there are three neighbors of each type, the theoretical modulation pattern (the bottom curve) is the product of the curves for each neighbor type raised to the third power.

computations.

Spin echoes at $9.25 \mathrm{GHz}$ were observed in dilute ruby at liquid-helium temperatures with the magnetic field applied essentially parallel to the optic axis of the crystal. ${ }^{4}$ The echoes could only be seen for the $\left(+\frac{1}{2} \longrightarrow-\frac{1}{2}\right)$ transition at $3.3 \mathrm{kG}$; no echoes could be seen for the $\left(+\frac{1}{2} \hookrightarrow+\frac{3}{2}\right.$ low-field $)$ transition at $0.8 \mathrm{kG}$, and we were unable to look for the echoes of the ( $+\frac{1}{2} \hookleftarrow+\frac{3}{2}$ high-field) transition as our magnet could not produce the required field. Our negative results for the echoes of the $\left(+\frac{1}{2}\right.$ $\rightarrow+\frac{3}{2}$ low-field) transition show that the low-field echo signals are less than $\frac{1}{400}$ of the signals for the echoes of the $\left(+\frac{1}{2} \rightarrow-\frac{1}{2}\right)$ transition with pulse separations from 1 to $2.5 \mu \mathrm{sec}$. Figures 4 and 5 show that the theoretical echo signals for the $\left(+\frac{1}{2} \rightarrow+\frac{3}{2}\right.$ low field) transition are of the order of $\frac{1}{200}$ of the signals for the echoes of the $\left(+\frac{1}{2}--\frac{1}{2}\right)$ transition. However, the theory includes no relaxation processes, and if the neglected relaxation processes are more important for the $\left(+\frac{1}{2}-+\frac{3}{2}\right)$ low-field) transition than for the $\left(+\frac{1}{2}--\frac{1}{2}\right)$ transition, the ratio of the echo signals from these two transitions would be smaller than theory predicts.

The amplitude of the echo signal for the $\left(+\frac{1}{2}\right.$ $--\frac{1}{2}$ ) transition was strongly modulated ${ }^{4}$ as $\tau$ was varied, and the modulation was quite dependent on the width of the excitation pulses. As shown in Fig. 8, the modulation of the echo envelope had much finer detail as the two pulses were made narrower. Increasing the width of the two excitation pulses seemed to merely smear out the details of the short-pulse modulation pattern in a relatively smooth manner. No basic changes in the pattern occurred other than loss of detail as the pulse widths were increased. To rigorously satisfy the short-pulse condition, the width of the excitation pulses must be small compared to the shortest precession period of the nuclear neighbors. However, for excitation pulses of 100 nsec which were approximately one-half the fastest nuclear period the pattern became relatively insensitive to further reductions in pulse width. Thus, the rigorous shortpulse condition seems too stringent. In Fig. 1, experiment and theory agree quite well for the location of the maxima and minima in the modulation patterns. The agreement is especially important since there are no adjustable parameters in the theory. Experiment (Fig. 8) and theory roughly agree for pulse separations between 2.5 and 3.5 $\mu$ sec but not in detail. This breakdown of agreement as the pulse separation is increased is to be expected, because as the pulse separation is increased the separation corresponds to more and more nuclear periods and small differences between the correct precession frequencies and those put in the theory lead to major discrepencies. The modulation patterns do not agree for the relative

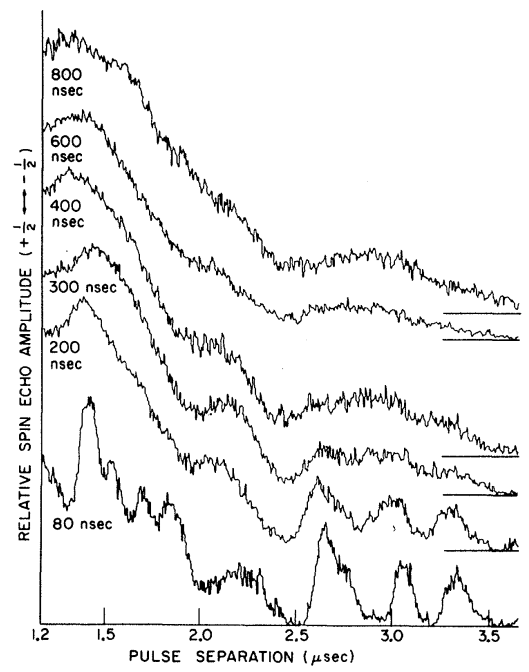

FIG. 8. Measured amplitude of the spin echoes in ruby [for the $\left(+\frac{1}{2} \longleftrightarrow-\frac{1}{2}\right)$ transition at $9.25 \mathrm{GHz}$ with $\theta=0$ ] versus the separation between the two excitation pulses for different durations of the pulses; the two pulses have the same width. 
strengths of the echoes. One reason for this disagreement is that the strengths of the relative maxima for the echo modulation pattern depend on the $B_{j}$ parameters, which have an uncertainty of approximately $10 \%$. Also the theoretical calculations neglect the $\mathrm{Cr}^{3+}-\mathrm{Cr}^{3+}$ interactions and the $\mathrm{Al} \leftarrow \mathrm{Al}$ interactions. There are no relaxation mechanisms in the theory.

As shown in Fig. 9, the echo signals rapidly disappeared as $\theta$ was increased. For the data of Fig. 9, the magnetic field was readjusted for resonance at each different angular setting, because the position of the line shifts when $\theta$ is changed. The echoes could no longer be seen when $\theta$ was greater than $3^{\circ}$, which corresponded to a reduction in the echo signal of about 100. A comparison of the experimental results of Fig. 9 with the theoretical predictions of Fig. 5 shows that the observed echoes are not as dependent on $\Theta$ as theory predicts. This reduction in the $\theta$ dependence is mainly due to the optic axis wandering (of the order of $1^{\circ}$ ) in our crystals which tends to smear out the sharp $\theta$ behavior.

\section{PHOTON ECHOES IN RUBY}

\section{A. Theory}

The photon echoes are associated with the various allowed transitions between the Zeeman components (shown in Fig. 10) of the $\bar{E}\left({ }^{2} E\right.$ ) excited state and the ${ }^{4} A_{2}$ ground state of the $\mathrm{Cr}^{3+}$ ion. The photon-echo behavior due to the interaction between

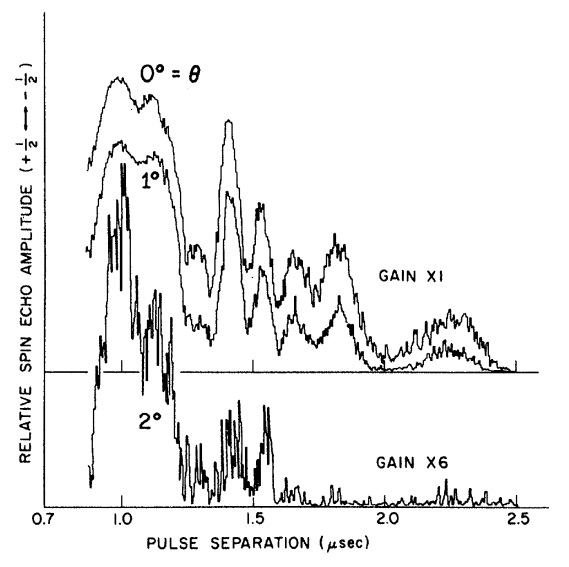

FIG. 9. Measured amplitude of the spin echoes in ruby versus the separation between the two excitation pulses as the angle $\theta$ between the optic axis and the magnetic field of approximately $3.3 \mathrm{kG}$ is increased. The two excitation pulses had equal widths of approximately $60 \mathrm{nsec}$. Note that for the $\theta=2^{\circ}$ curve, the gain has been increased by 6 .

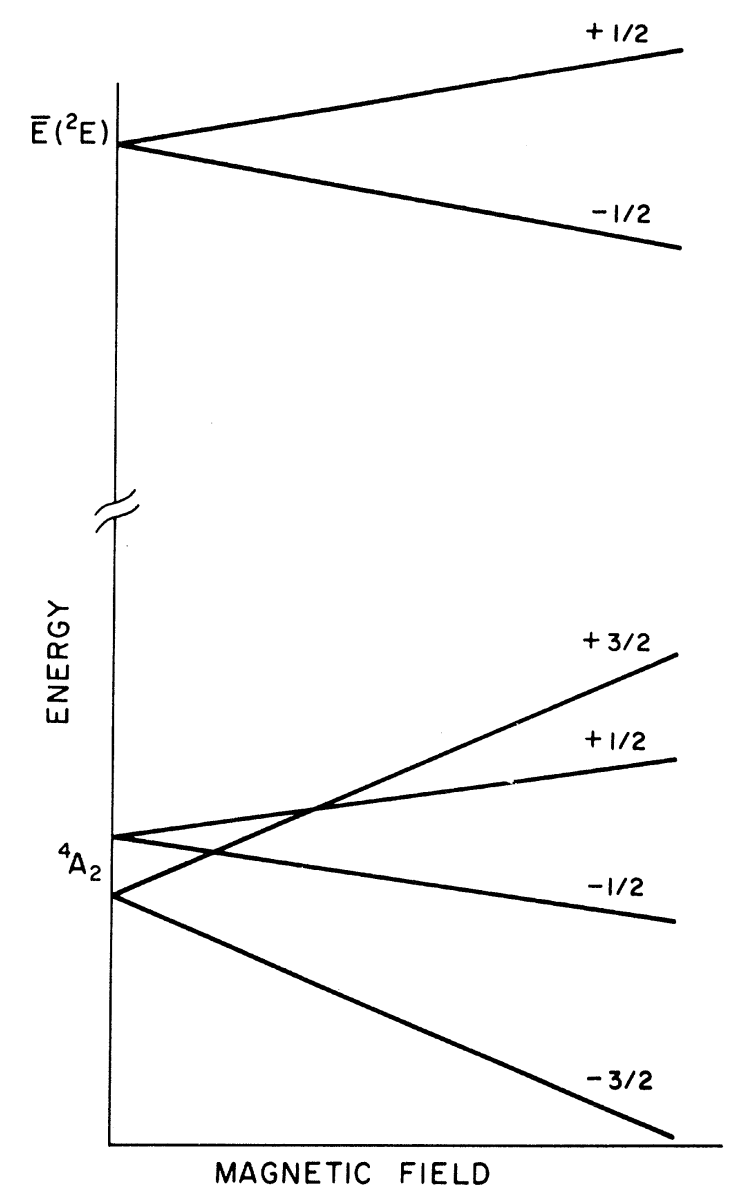

FIG. 10. Splitting of the $\bar{E}\left({ }^{2} E\right)$ excited state and the ${ }^{4} A_{2}$ ground state of the $\mathrm{Cr}^{3+}$ ion versus the strength of the magnetic field applied along the optic axis.

the $\mathrm{Cr}^{3+}$ ions and the $\mathrm{Al}$ nuclear neighbors will be obtained for the echoes associated with transitions between any two Zeeman components designated as 1 and 2, where 1 is a component of the $\bar{E}\left({ }^{2} E\right)$ excited state and 2 is a component of the ${ }^{4} A_{2}$ ground state. For simplicity the crystal size is assumed small compared to $\lambda^{3}$ (the wavelength of the resonant radiation). The calculation is easily extended to large samples, but the results, except for directional effects, are the same. ${ }^{10}$

The Hamiltonian $\mathcal{F}$ for the $\mathrm{Cr}^{3+}$ ion and the $\mathrm{Al}$ nuclear neighbors has the form of Eq. (2.1), and for photon echoes $\mathfrak{F}^{0}$ can be split up into the two terms

$$
\mathfrak{H}^{0}=\mathfrak{H}_{E}^{0}+\mathcal{H}_{S}^{0}
$$

$\mathcal{H}_{E}^{0}$ contains the major terms of the Hamiltonian for the $\mathrm{Cr}^{3+}$ ion and is diagonalized to yield the ${ }^{4} A_{2}$ ground state and the first excited state $\bar{E}\left({ }^{2} E\right)$. The interaction of the $\mathrm{Cr}^{3+}$ ion with $\overrightarrow{\mathrm{H}}$ is included in $\mathcal{H}_{S}^{0}$ 
which can be treated as a small perturbation. The application of $\overrightarrow{\mathrm{H}}$ splits the $\bar{E}\left({ }^{2} E\right)$ level into the two Zeeman components $\psi\left({ }^{2} E,+\frac{1}{2},-1\right)$ and $\psi\left({ }^{2} E,-\frac{1}{2}\right.$, +1 ), where $\pm \frac{1}{2}$ is the spin quantum number and \pm 1 is an orbital quantum number which describes the rotation properties of the wave functions about the optic axis of the crystal. ${ }^{15,16}$ The perturbation of the excited state caused by $\mathfrak{F}_{S}^{0}$ can be represented by the spin Hamiltonian ${ }^{17}$

$$
\mathfrak{H}_{S}^{1}=g_{\|} \mu_{\beta} H_{z} S_{z}+g_{\perp} \mu_{\beta}\left(H_{x} S_{x}+H_{y} S_{y}\right),
$$

where the $z$ axis is the optic axis of the crystal, the effective spin is $\frac{1}{2}$, and

$$
g_{11}=2.445 \text { and } g_{\perp} \leqslant 0.06 \text {. }
$$

Because we are only concerned with the situation where the angle $\theta$ is relatively small, Eq. (4.2) can be simplified to

$$
\mathfrak{F}_{S}^{1}=g_{\|} \mu_{\beta} H_{z} S_{z},
$$

which is diagonal. The effect of $\mathcal{F}_{S}^{0}$ on the ${ }^{4} A_{2}$ ground state is given by the spin Hamiltonian used earlier in the spin-echo calculation.

Since the echo signal is proportional to the radiated power, the intensity of the photon echo is proportional to $\langle\overrightarrow{\mathrm{P}}(t)\rangle^{2}$ of Eq. (2.22), where $\overrightarrow{\mathrm{P}}_{12}$ is the matrix element of the electric-dipole moment operator $\overrightarrow{\mathrm{P}}$ between the Zeeman components 1 and 2 involved in the echo formation. For evaluation of the $\operatorname{Tr} C_{j}$ we assume that the terms $h_{1,2}^{j}$ have the form of Eq. (3.4) for both the excited and ground states of the ion, but that the interaction parameters $A_{j}^{*}$ and $B_{j}^{*}$ (values unknown) for the excited state are different from those of the ground state, $A_{j}$ and $B_{j}$, which are known. Because each of the Zeeman components of the excited state has a different orbital function, $A_{j}^{*}$ and $B_{j}^{*}$ are assumed to be different for each component. $\vec{S}_{11}$ is considered to be $\pm \frac{1}{2} \overrightarrow{\mathrm{e}}_{3}$ according to whether $\psi\left({ }^{2} E, \pm \frac{1}{2}, \mp 1\right)$ is designated as state 1 . With these considerations, $\operatorname{Tr} C_{j}$ is given by the result of the spin-echo calculation.

In order to get some familiarity with the predictions of the theory and to keep the problem manageable, the following simplifications are made. As the separation between the two excitation pulses is short compared to the frequencies associated with the electric quadrupole interaction term, this term is neglected by setting $Q^{j}=0 . \quad B_{j}^{*}$ is assumed to be given by the dipolar interaction as

$$
B_{j}^{*}=g_{11} \mu_{\beta} \mu_{N} g_{n} \text {. }
$$

$g_{n}=1.456$ for $\mathrm{Al}^{27}$, and $\mu_{N}$ is the nuclear magneton. $A_{j}^{*}$ is assumed to be proportional to $A_{j}$ with the same constant of proportionality for all the neighbors, i. e.,

$$
A_{j}^{*}=A_{j}\left(A^{*} / A\right)
$$

and in the theoretical curves, $A^{*} / A$ is the only adjustable parameter. Because $Q_{j}=0$ in the computations, the $\Pi_{j} \operatorname{Tr} C_{j}$ is real; all the theoretical curves of this section are proportional to the square of $\Pi_{j} \operatorname{Tr} C_{j}$.

\section{B. Results}

The results are presented for the theoretical dependence of the intensity of the photon echoes on the angle $\theta$ between the applied magnetic field $\overrightarrow{\mathrm{H}}$ and the optic axis ( $\theta$ dependence), on $\vec{H}(\vec{H}$ dependence), and on the separation $\tau$ between the two excitation pulses ( $\tau$ dependence). We are primarily interested in the theoretical behavior for the $\left(+\frac{1}{2}-+\frac{1}{2}\right) \sigma^{-}$and $\left(-\frac{1}{2} \leftarrow-\frac{1}{2}\right) \sigma^{+}$transitions because the $\sigma^{ \pm}$photon echoes have been studied experimentally. ${ }^{9-12,18}$ The experiments used linearly polarized excitation radiation, and the observed signal was due to both $\sigma^{+}$and $\sigma^{-}$echoes of unknown relative intensity. Thus, a precise comparison with theory is impossible; however, the data are very useful for setting limits on the theoretical parameters. Because the theoretical $\overrightarrow{\mathrm{H}}$ and $\Theta$ dependence of the $\sigma^{+}$and $\sigma^{-}$ echoes are qualitatively similar, only the $\sigma^{+}$results are presented; both transitions are considered when the $\tau$ dependence is discussed. The $\theta$ dependence of theory and experiment are compared in Figs. 11 and 12. For these curves, $H_{z}$, the component of $\overrightarrow{\mathrm{H}}$ along the optic axis, was kept constant, and $\theta$ was varied by increasing the transverse component in order to duplicate the conditions of the experiment. ${ }^{10}$ Each curve was independently normalized to unity at $\theta=0$. An important feature of these curves is that values of the interaction parameter $A * / A$ of the order of unity give reason-

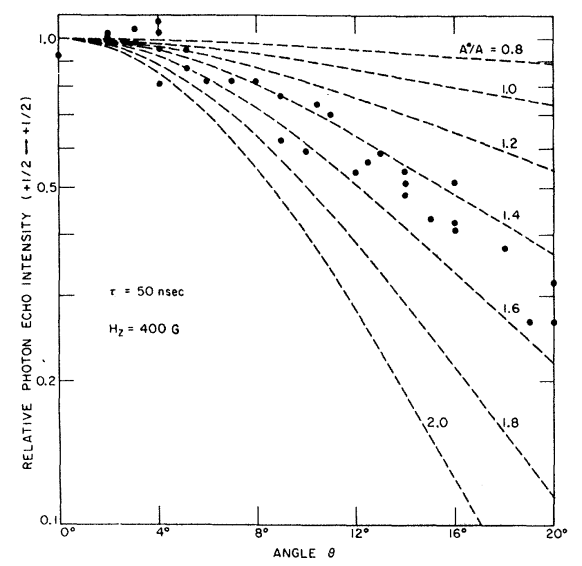

FIG. 11. Comparison of theory for the $\left(+\frac{1}{2} \longleftrightarrow+\frac{1}{2}\right)$ transition and experiment for the intensity of the photon echoes in ruby with a pulse separation of $50 \mathrm{nsec}$ versus the angle between the optic axis and the applied magnetic field. Data of Abella, Kurnit, and Hartmann (Ref. 10). 


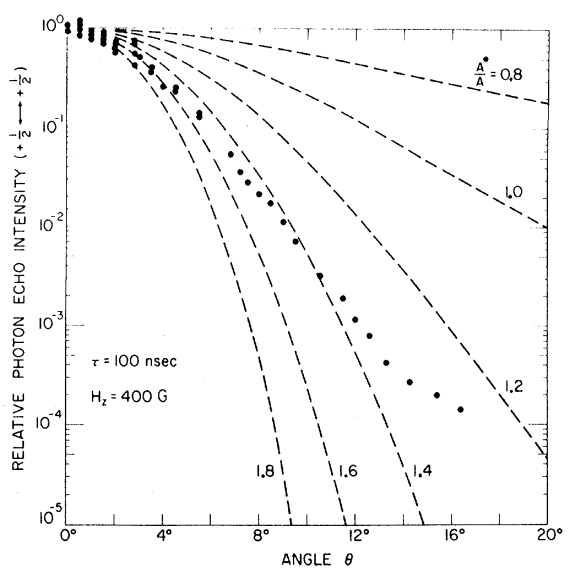

FIG. 12. Comparison of theory for the $\left(+\frac{1}{2} \longleftrightarrow+\frac{1}{2}\right)$ transition and experiment for the intensity of the photon echoes in ruby with a pulse separation of $100 \mathrm{nsec}$ versus the angle between the optic axis and the applied magnetic field. Data of Abella, Kurnit, and Hartmann, (Ref. 10).

able agreement with experiment. Changing $\tau$ from 50 to 100 nsec causes a dramatic change in the $\theta$ dependence, but $A^{*} / A=1.4$ gives acceptable agreement with experiment in both cases. Thus, the $\theta$ dependence of the photon echoes seems to be caused by the interactions between the $\mathrm{Cr}^{3+}$ ion and the $\mathrm{Al}$ nuclear neighbors and to be understandable within the framework of the general theory. Using $A * / A$ $=1.4$, which best fits the angular data, we refer to Figs. 13 and 14 (the remaining curves in this section, including Figs. 13 and 14, have been normalized to unity for $\Theta=0$ ) and predict that the $\overrightarrow{\mathrm{H}}$ dependence for $\Theta=0$ should be negligible when $\tau$ is less than 100 nsec and should be observable when $\tau$ is of the order of $200 \mathrm{nsec}$. At first sight these predictions seem to be at variance with tha data. ${ }^{11}$ However, if one remembers that the above theory is a high-field theory which neglects low-field processes such as mutual spin flips between the $\mathrm{Cr}^{3+}$ ion and the $\mathrm{Al}$ neighbors, the agreement can be considered acceptable; the observed sharp $\overrightarrow{\mathrm{H}}$ dependence is due to the low-field processes. Another feature of the data for the field dependence is the sharp dips at 2 and $4 \mathrm{kG}$, which correspond to the energy level crossings in the ${ }^{4} A_{2}$ ground state of the $\mathrm{Cr}^{3+}$ ion. For $\Theta \neq 0$, theory predicts a marked reduction in echo intensity for the $\sigma^{+}$transition at the 2-kG energy-level crossing and for the $\sigma^{-}$transition at the $4-\mathrm{kG}$ crossing, but for $\theta=0$, these theoretical dips do not appear. Because the observed echoes were due to echoes from both transitions, two dips would be expected. Figure 14 shows that increasing $\overrightarrow{\mathrm{H}}$ does not, in general, lead to more intense echoes, and actual reductions in intensity can occur depending on the values of the

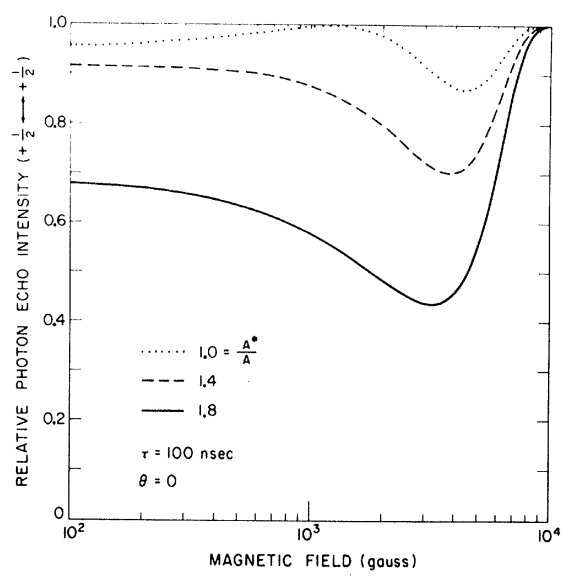

FIG. 13. Theoretical dependence of the intensity of the photon echoes in ruby of the $\left(+\frac{1}{2} \longleftrightarrow+\frac{1}{2}\right)$ transition with a pulse separation of $100 \mathrm{nsec}$ on the strength of the magnetic field applied along the optic axis.

interaction constants between the $\mathrm{Cr}^{3+}$ ion and the nuclear neighbors. However, in the limit of ex-

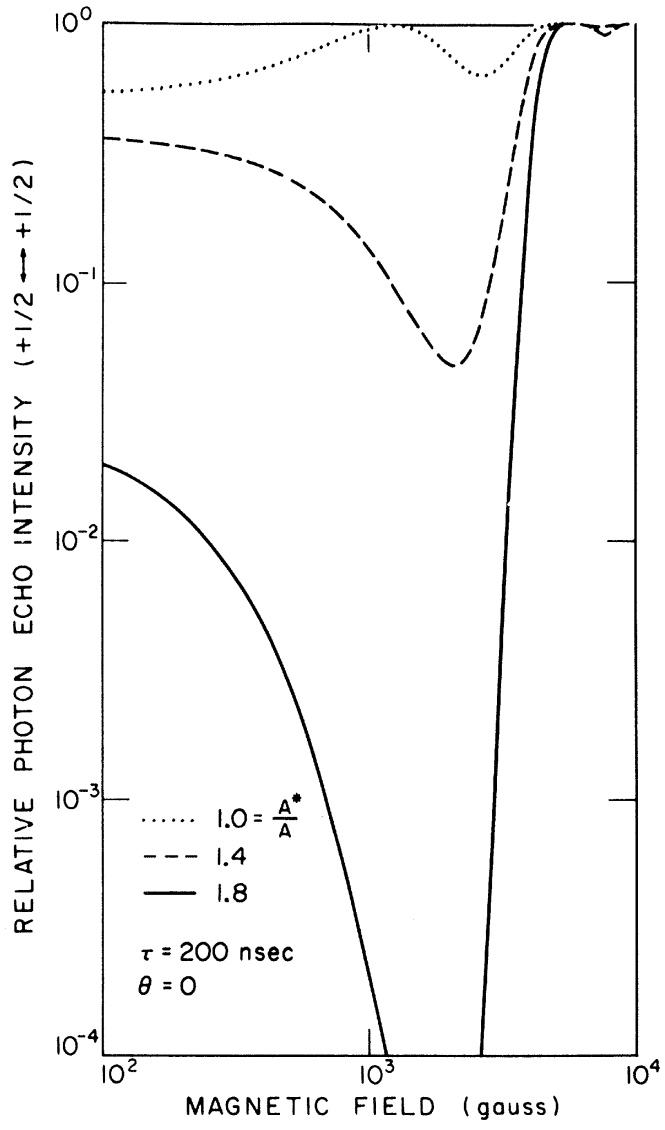

FIG. 14. Theoretical dependence of the intensity of the photon echoes of the $\left(+\frac{1}{2} \longleftrightarrow+\frac{1}{2}\right)$ transition with a pulse separation of $200 \mathrm{nsec}$ on the strength of the magnetic field applied along the optic axis. 


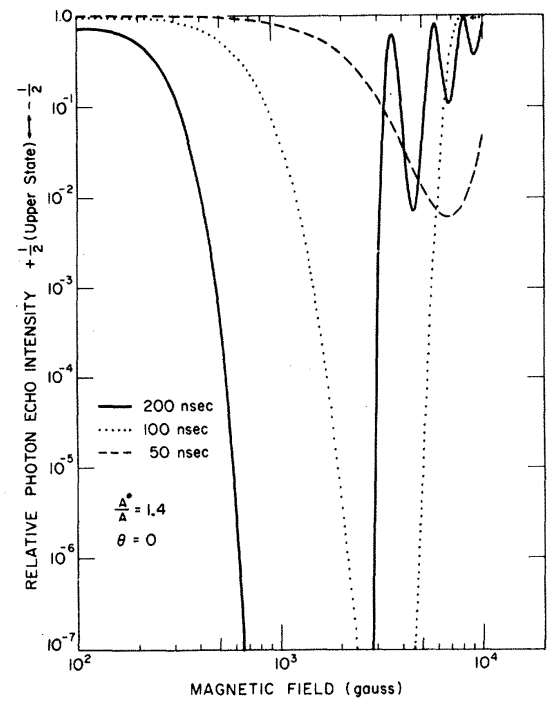

FIG. 15. Theoretical dependence of the intensity of the photon echoes in ruby of the $\left(+\frac{1}{2}\right.$ upper state $\left.\longleftrightarrow-\frac{1}{2}\right)$ transition on the strength of the magnetic field applied along the optic axis.

tremely high fields, higher than $10 \mathrm{kG}$, the influence of the nuclei becomes negligible.

Photon echoes associated with the $\left(+\frac{1}{2}\right.$ upper state $\left.--\frac{1}{2}\right)$ and $\left(-\frac{1}{2}\right.$ upper state $\left.-+\frac{1}{2}\right) \pi$ transitions have been seen for short pulse separations, ${ }^{11}$ but no detailed data are yet available. The theoretical results are much more striking for the $\pi$ echoes. The predicted $\overrightarrow{\mathrm{H}}$ and $\tau$ dependence for the $\left(+\frac{1}{2}\right.$ upper state $\left.--\frac{1}{2}\right)$ echoes are shown in Fig. 15 when $\Theta=0$, and the marked, orders-of-magnitude, $\overrightarrow{\mathrm{H}}$ dependence with $\tau$ of the order of $100 \mathrm{nsec}$ should be easily observable. Figure 16 shows the predicted dynamic $\tau$ dependence of these echoes with $\Theta=0$ and $\overrightarrow{\mathrm{H}}=3 \mathrm{kG}$.

Photon echoes due to both $\sigma^{+}$and $\sigma^{-}$transitions have been observed as a function of $\tau$ with $\overrightarrow{\mathrm{H}} \approx 3 \mathrm{kG}$ nearly parallel to the optic axis; the echo intensity was modulated as $\tau$ was varied, and the modulation dramatically increased as $\theta$ was increased. ${ }^{18}$ As shown in Fig. 17 for the $\sigma^{-}$transition with $\theta=0$ and $\overrightarrow{\mathrm{H}}=3 \mathrm{kG}$, theory predicts substantial modulation with $A^{*} / A=1.4$, and Fig. 18 shows that, if $\Theta$ is increased to only $1^{\circ}$, the photon echo is essentially unobservable for $\tau$ between 200 and $300 \mathrm{nsec}_{\text {。 }}$ However, for the $\sigma^{+}$transition, the theoretical behavior is quite different. Using the value of $A^{*} / A$ $=0.8$ for this transition which best fits the angular fall-off data of Figs. 11 and 12, we obtain Fig. 19 for the $\Theta$ and $\tau$ dependence of the $\sigma^{+}$photon echoes. Clearly, more experimental work using circularly polarized excitation pulses is needed, and such photon-echo experiments are in progress to test the main features of the theory and to gain infor-

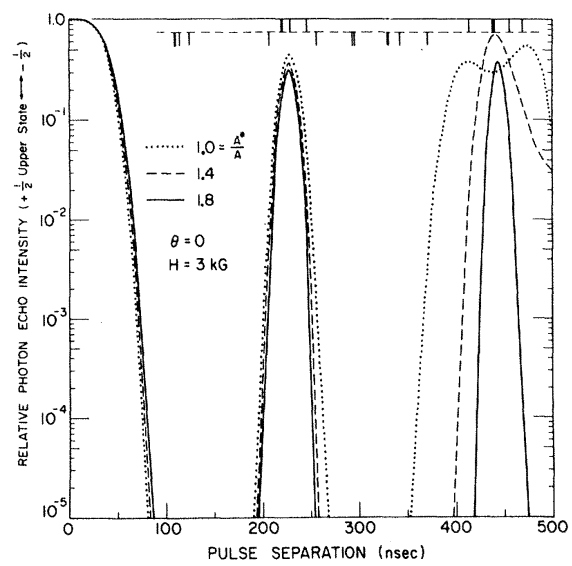

FIG. 16. Theoretical dependence of the intensity of

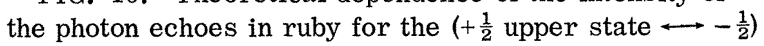
transition on the separation $\tau$ between the two excitation pulses with different values of the interaction parameter $A * / A$. The short vertical lines below the dashed horizontal line (relative minima for the simple model) mark the values of $\tau$ equal to an integral multiple plus one-half of the Larmor periods of the nuclear neighbors with $Q^{j}=0$ and $A^{*} / A=1$. 4; the vertical lines above the dashed line (relative maxima for the simple model) mark the values of $\tau$ equal to integral multiples of the Larmor periods.

mation about the interaction constants $A_{j}^{*}$ and $B_{j}^{*}$.

\section{SIMPLE MODEL}

Electron-spin and photon echoes are generally

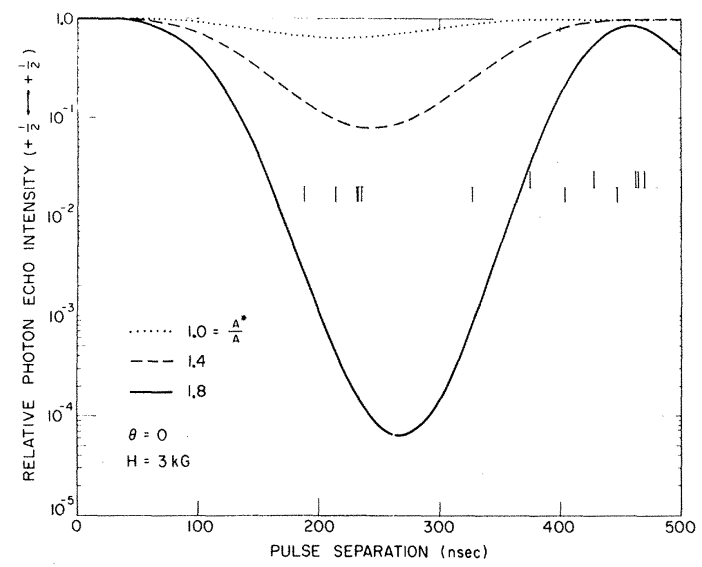

FIG. 17. Theoretical dependence of the intensity of the photon echoes in ruby for the $\left(+\frac{1}{2} \longleftrightarrow+\frac{1}{2}\right)$ transition on the separation $\tau$ between the two excitation pulses with different values of the interaction parameter $A^{*} / A$. The short vertical lines below the horizontal line (relative minima for the simple model) mark the values of $\tau$ equal to an integral multiple plus one-half of the Larmor periods of the nuclear neighbors with $\mathrm{Q}^{j}=0$ and $A * / A$ $=1.8$; the vertical lines above the line (relative maxima for the simple model) mark the values of $\tau$ equal to integral multiples of the Larmor periods. 


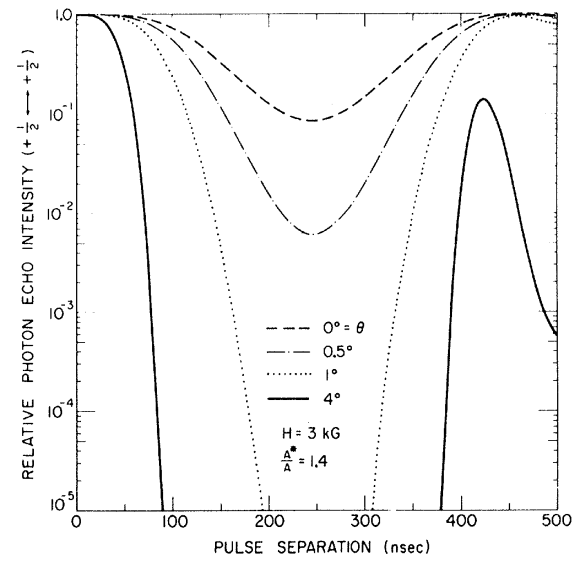

FIG. 18. Theoretical dependence of the intensity of the photon echoes for the $\left(+\frac{1}{2} \longleftrightarrow+\frac{1}{2}\right)$ transition on the separation between the two excitation pulses and on the angle $\theta$ between the magnetic field of $3 \mathrm{kG}$ and the optic axis.

produced by the successive excitation of an ensemble of echo atoms by two intense resonant excitation pulses. In the simplest case, the first pulse induces a precessing macroscopic dipole moment which soon decays because the dipole moments of the individual echo atoms precess at slightly different frequencies because of their different local environments. It is well known ${ }^{19,20}$ that the second pulse (considered to be a $180^{\circ}$ pulse) negates the relative phase of these precessing dipoles. If the local environment of each echo atom is constant, the dipoles, after the second pulse, will rephase at the same rate at which they had dephased, and the macroscopic moment will reform to produce the echo. To the extent that the local environments fluctuate in time, the rephasing is incomplete, and as the pulse separation is increased an exponential decay of the echo amplitude is usually observed. The surprising behavior of the spin and photon echoes from isolated echo atoms in ionic crystals is that in many cases the echo amplitude oscillates between quite large and small values as the pulse separation is increased. This echo modulation is caused by the slow (compared to the precession frequency of the dipoles) regular time dependence of the local environments of the echo atoms, which is due to the precession of the magnetic nuclei of the neighboring atoms.

The excitation pulses are assumed to couple together only the two echo states of the echo atom, and for an understanding of the echo behavior, the echo atom can be considered as a simple two-level system. The echo states are designated as states 1 and 2, where state 1 has the higher energy. The time dependence of the local environment due to the precession of the nuclear neighbors of the echo atom causes the energy separation $\omega(t)$ between the two echo states to be time dependent. $\omega(t)$ can be separated into the two terms

$$
\omega(t)=\omega_{0}+\delta(t)
$$

$\delta(t)$ describes the effect of the neighbors on the echo atom and has a time-dependent part which is responsible for the dephasing; $\omega_{0}$ is the constant energy separation between the two echo states in the absence of the neighboring nuclei. $\delta(t)$ is usually very small compared to $\omega_{0}$; for photon echoes $\delta(t)$ $\approx 10^{-8} \omega_{0}$ and for electron-spin echoes $\delta(t) \approx 10^{-4} \omega_{0}$.

Consider the echo atom to interact with only one nuclear neighbor which has no electric quadrupole moment, and let the Hamiltonain for the echo atom and the neighbor be given by

$$
\begin{aligned}
\mathcal{H}= & =\frac{1}{2} \hbar \omega_{0} \sigma_{3}+A \overrightarrow{\mathrm{S}} \cdot \overrightarrow{\mathrm{I}} \\
& +B \overrightarrow{\mathrm{S}} \cdot\left[3 \overrightarrow{\mathrm{r}}(\overrightarrow{\mathrm{r}} \cdot \overrightarrow{\mathrm{I}}) / r^{5}-\overrightarrow{\mathrm{I}} / r^{3}\right]-\hbar \gamma \overrightarrow{\mathrm{H}} \cdot \overrightarrow{\mathrm{I}} .
\end{aligned}
$$

$\overrightarrow{\mathrm{S}}$ and $\overrightarrow{\mathrm{I}}$ are the spin operators for the echo atom and the neighbor, respectively。 $\overrightarrow{\mathrm{H}}$ is the applied magnetic field, and $\vec{r}$ is the vector from the atom to the neighbor. Equation (5.2) can be rewritten in the simpler form

$$
\mathcal{H}=\frac{1}{2} \hbar \omega_{0} \sigma_{3}-\hbar \gamma \overrightarrow{\mathrm{H}}_{\epsilon} \cdot \overrightarrow{\mathrm{I}},
$$

where $\overrightarrow{\mathrm{H}}_{\epsilon}$ is an operator that describes an effective magnetic field ${ }^{8}$ at the neighbor site. Define the two quantities

$$
\overrightarrow{\mathrm{H}}_{\epsilon}^{1} \equiv\left\langle 1\left|\overrightarrow{\mathrm{H}}_{\epsilon}\right| 1\right\rangle \text { and } \overrightarrow{\mathrm{H}}_{\epsilon}^{2} \equiv\left\langle 2\left|\overrightarrow{\mathrm{H}}_{\epsilon}\right| 2\right\rangle \text {. }
$$

$|1\rangle$ and $|2\rangle$ are the eigenfunctions for the echo states 1 and 2. $\overrightarrow{\mathrm{H}}_{\epsilon}^{1}$ or $\overrightarrow{\mathrm{H}}_{\epsilon}^{2}$ is the effective field for the nuclear neighbor when the echo atom is in state 1 or 2 , respectively.

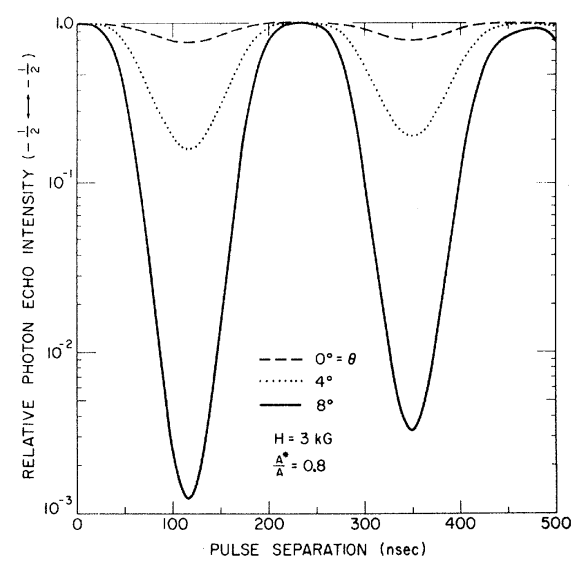

FIG. 19. Theoretical dependence of the intensity of the photon echoes for the $\left(-\frac{1}{2} \longleftrightarrow-\frac{1}{2}\right)$ transition on the separation between the two excitation pulses and on the angle $\theta$ between the magnetic field of $3 \mathrm{kG}$ and the optic axis. 
tively. Because the second term in Eq. (5.3) is very small compared to the first, the expression for $\omega(t)$ can be immediately written

$$
\hbar \omega(t)=\langle 1|\mathfrak{H C}| 1\rangle-\langle 2|\mathfrak{H C}| 2\rangle,
$$

which is equivalent to

$$
\omega(t)=\omega_{0}-\gamma\left[\overrightarrow{\mathrm{H}}_{\epsilon}^{1}-\overrightarrow{\mathrm{H}}_{\epsilon}^{2}\right] \cdot \overrightarrow{\mathrm{I}}(t) \quad .
$$

The simple model treats the nuclear spin vector $\overrightarrow{\mathrm{I}}(t)$ as a classical quantity. Thus, once the motion of $\overrightarrow{\mathrm{I}}(t)$ is known, Eq. (5.6) allows the echo behavior due to the interaction between the echo atom and the nuclear neighbor to be calculated. If the echo atom were in either echo state 1 or 2 , the motion of $\sharp(t)$ would be a precession about $\overrightarrow{\mathrm{H}}_{\epsilon}^{1}$ or $\overrightarrow{\mathrm{H}}_{\epsilon}^{2}$. When the echo atom is in a linear superposition of states (partially in state 1 and partially in state 2), calculation of the expectation value of $\vec{I}$ gives a result that corresponds to the vector summation of two precessing vectors, where one vector precesses about $\overrightarrow{\mathrm{H}}_{\epsilon}^{1}$ with angular frequency $\omega_{1}$ and the other precesses about $\overrightarrow{\mathrm{H}}_{\epsilon}^{2}$ with angular frequency $\omega_{2}$. Thus, when the echo atom is in a linear superposition of states we write

$$
\overrightarrow{\mathrm{I}}(t)=\overrightarrow{\mathrm{I}}_{1}(t)+\overrightarrow{\mathrm{I}}_{2}(t) \text {, }
$$

where we interpret $\overrightarrow{\mathrm{I}}_{1}(t)$ as precessing about $\overrightarrow{\mathrm{H}}_{6}^{1}$ with angular frequency $\omega_{1}$ and $\overrightarrow{\mathrm{I}}_{2}(t)$ as precessing about $\overrightarrow{\mathrm{H}}_{\epsilon}^{2}$ with frequency $\omega_{2}$ :

$$
\omega_{1}=\gamma\left|\overrightarrow{\mathrm{H}}_{\epsilon}^{1}\right| \text { and } \omega_{2}=\gamma\left|\overrightarrow{\mathrm{H}}_{\epsilon}^{2}\right| \text {. }
$$

If the echo atom is equally in states 1 and 2 , the magnitudes of $\overrightarrow{\mathrm{I}}_{1}(t)$ and $\overrightarrow{\mathrm{I}}_{2}(t)$ are the same. This description is certainly correct when the echo atom is completely in state 1 or 2 and is the simplest behavior we can ascribe to the nuclear spin vector to explain the observed modulated echo behavior in an intuitive way. Referring to Eqs. (5.1) and (5.6), we see that in terms of the simple model, the time dependence of $\delta(t)$ is given by two sinusoidal components, one oscillating at $\omega_{1}$ and the other at $\omega_{2}$. As the time-dependent parts of $\vec{I}_{1}(t)$ and $\vec{I}_{2}(t)$ are orthogonal to $\overrightarrow{\mathrm{H}}_{\epsilon}^{1}$ and $\overrightarrow{\mathrm{H}}_{\epsilon}^{2}$, respectively, only the projections of the time-dependent parts of $\overrightarrow{\mathrm{I}}_{1}(t)$ on $\overrightarrow{\mathrm{H}}_{\epsilon}^{2}$ and $\overrightarrow{\mathrm{I}}_{2}(t)$ on $\overrightarrow{\mathrm{H}}_{\epsilon}^{1}$ are nonzero, and consequently, evaluation of Eq. (5.6) shows that the amplitudes of the two sinusoidal components of $\delta(t)$ are proportional to the sine of the angle $\Delta$ between $\overrightarrow{\mathrm{H}}_{\epsilon}^{1}$ and $\overrightarrow{\mathrm{H}}_{\epsilon}^{2}$. These results are easily extended to an echo atom with more than one nuclear neighbor. For this situation, $\delta(t)$ is again given by a sum of sinusoidal components with two components for each neighbor, and the amplitudes of the components for the $j$ th neighbor are proportional to the sine of the angle $\Delta_{j}$ between the two effective fields for the $j$ th neighbor.

The fact that $\delta(t)$ is given by a sum of sinusoidally oscillating components allows for the partial re- phasing that is necessary to obtain the spin or photon echoes. The most general condition for the occurrence of the two-pulse echo is that the integrals of $\delta(t)$ with respect to time after each of the two excitation pulses be the same for all the echo atoms. This requirement will be satisfied for a single oscillating component of $\delta(t)$, independent of the phase of the oscillation (the phase varies randomly from echo atom to echo atom) when the separation between the two pulses is equal to an integral multiple of the period of the oscillation. Usually $\delta(t)$ is composed of many components, and the rephasing condition cannot be satisfied for all of them simultaneously. Thus, only relative maxima in the echo signal are expected when the pulse separation is equal to integral multiples of the periods of the oscillating components. If the magnitude of $\delta(t)$ is increased, the dephasing effects of the components for which the rephasing condition is not satisfied will become much larger and the echosignals will be rapidly reduced.

The simple model can be applied to both electronspin and photon echoes in ruby, since the echo dephasing caused by the nuclear neighbors is independent of the energy separation $\omega_{0}$ between the two echo states if $\omega_{0}$ is large compared to the interaction energy between $\mathrm{Cr}^{3+}$ ion and the nuclear neighbors. However, the spin-echo signals are proportional to the macroscopic dipole moment of the system at $t=2 \tau$, and the photon echoes are proportional to the square of the macroscopic moment at $t$ $=2 \tau$. Thus, an equivalent amount of dephasing would appear much more intense for photon echoes than for spin echoes. The $\mathrm{Cr}^{3+}$ ion which is responsible for the echo radiation in both cases interacts quite strongly with the magnetic nuclei of the 13 nearest $\mathrm{Al}$ neighbors. ${ }^{8}$ If the angle $\theta$ between the applied magnetic field $\overrightarrow{\mathrm{H}}$ and the optic axis is zero, the angle $\Delta$ between the two effective fields for the nearest neighbor is zero, and this neighbor does not contribute to the echo dephasing. The remaining 12 neighbors form four groups each composed of three equivalent neighbors; all the members of a group dephase the echo in the same way. The strengths of the components for a group are proportional to $\sin \Delta$ for the equivalent nuclei of the group.

Because the Al nuclei have an electric-quadrupole moment, $\delta(t)$ contains so many components that application of the model for obtaining the echo envelope is difficult when the pulse separations are large compared to the period of the quadrupolar splitting (the spin-echo case). However, for photon echoes excited by pulses less than $0.5 \mu$ sec apart the problem could be simplified by neglecting the quadrupole splitting. For $\Theta=0 \delta(t)$ can then be resolved into eight sinusoidal components, where 
each group contributes two components, and in Figs. 16 and 17 the relative maxima and minima are easily observed.

When $\overrightarrow{\mathrm{H}}$ is tilted away from the optic axis, the 13 neighbors become inequivalent, but for relatively small values of $\theta$ the most important change is that as $\theta$ is increased $\delta(t)$ sharply increases. $\delta(t)$ has a relative minimum for $\overrightarrow{\mathrm{H}}$ parallel to the optic axis, because the $\Delta_{j}$ angles of the individual neighbors are smallest when $\theta=0$. As was noted earlier, ${ }^{4}$ in many cases the $\Delta_{j}$ angles increase much faster than $\theta$, as $\theta$ is increased. This feature explains the angular dependence of both spin and photon echoes. As $\theta$ is increased, $\delta(t)$ increases, and the echo signals are reduced. The reduction occurs regardless of the pulse separation, because the rephasing condition cannot be satisfied simultaneously for all the components. The dips observed in the photon-echo signals for $\overrightarrow{\mathrm{H}}=2$ and $4 \mathrm{kG}$ with $\Theta=0$ occur at the energy-level crossings in the ${ }^{4} A_{2}$ ground state of the $\mathrm{Cr}^{3+}$ ion. At these crossings $\delta(t)$ is a very sharp function of $\Theta$, and a small increase in $\theta$ leads to a large reduction in the echo signal. As the optic axis varies by about 1 degree throughout the crystal, reductions in the echo signals are expected at the crossings. In the theoretical results for the spin echoes, the very large difference between the predicted echo amplitudes of the $\left(+\frac{1}{2} \longleftrightarrow+\frac{3}{2}\right.$ low field $)$ and the $\left(+\frac{1}{2} \hookrightarrow+\frac{3}{2}\right.$ high field) transitions is due to the difference between the $\Delta_{j}$ angles for the two field values. The $\Delta_{j}$ angles are much smaller for the high-field transition than for the low-field transition. Similarly, for the photon echoes the marked difference between the theoretical behavior of the echoes of the $\left(+\frac{1}{2} \leftarrow+\frac{1}{2}\right)$ transition and the $\left(+\frac{1}{2} \longleftrightarrow-\frac{1}{2}\right)$ transition is mainly due to the $\Delta_{j}$ angles being much smaller for the $\left(+\frac{1}{2} \longrightarrow+\frac{1}{2}\right)$ transition than for the $\left(+\frac{1}{2} \longrightarrow-\frac{1}{2}\right)$ transition.

\section{ACKNOWLEDGMENTS}

We wish to thank Larry Levin, Norman Kurnit, and Paul Liao for interesting and valuable discussions.

\footnotetext{
*Work supported in part by the Joint Services Electronics Program (U. S. Army, Navy, and Air Force), under Contract No. DA-28-043 AMC-00099(E) and in part by the Army Research Office, Durham, under Contract No. DA-31-124-ARO-D-341.

${ }^{\dagger}$ Present address: IBM Watson Research Center, Yorktown Heights, N. Y. 10598.

\$Alfred P. Sloan Research Fellow.

${ }^{1}$ W. B. Mims, K. Nassau, and J. D. McGee, Phys. Rev. 123, 2059 (1961).

${ }^{2}$ J. A. Cowen and D. E. Kaplan, Phys. Rev. 124, 1098 (1961).

${ }^{3}$ L. G. Rowan, E. L. Hahn, and W. B. Mims, Phys. Rev. 137, A61 (1965).

${ }^{4}$ D. Grischkowsky and S. R. Hartmann, Phys. Rev. Letters 20, 41 (1968).

${ }^{5}$ A. K. Saha and T. P. Das, Theory and Applications of Nuclear Induction (Saha Institute of Nuclear Physics, Calcutta, India, 1957).

${ }^{6}$ A. Abragam, The Principles of Nuclear Magnetism (Oxford U. P., London, England, 1969).

${ }^{7}$ G. M. Zhidomirov and K. M. Salikov, Teor. i Eksperim. Khim. 4, 514 (1968).

${ }^{8}$ N. Laurance, E. C. McIrvine, and J. Lambe, J。 Phys. Chem. Solids 23, 515 (1962).

${ }^{9}$ N. A. Kurnit, I. D. Abella, and S. R. Hartmann, Phys. Rev. Letters 13 , 567 (1964).

${ }^{10}$ I. D. Abella, N. A. Kurnit, and S. R. Hartmann, Phys. Rev. 141, 391 (1966).
}

\footnotetext{
${ }^{11}$ N. A. Kurnit and S. R. Hartmann, in Interaction of Radiation with Solids (Plenum, New York, 1967), pp. 693-701.

${ }^{12}$ A. Compaan, L. Q. Lambert, and I. D. Abella, Phys. Rev. Letters 20, 1089 (1968).

${ }^{13}$ For photon echoes, $\omega_{0}$ is approximately $10^{8}$ times larger than the nuclear frequencies and the neglect of $H_{12}^{I}$ and $H_{21}^{I}$ is always correct. For electron-spin echoes, if the strength of $\overrightarrow{\mathrm{H}}$ is sufficient to put $\omega_{0}$ in the microwave range, in most cases $\omega_{0}$ is of the order of $10^{4}$ times larger than the nuclear frequencies, and $H_{12}^{I}$ and $H_{21}^{I}$ can be neglected. However, for spin echoes, if $\vec{H}$ is small, $\omega_{0}$ can be of the order of the nuclear frequencies, and $H_{12}^{I}$ and $H_{21}^{I}$ must be retained; in the following work we assume $\overrightarrow{\mathrm{H}}$ is large enough so that neglect of $H_{12}^{I}$ and $H_{21}^{I}$ is valid.

${ }^{13 a}$ The arbitrary phase $2 \tau \omega_{0}$ is determined by the as sumed form of $R_{1}$ and $R_{2}$.

${ }^{14}$ E. O. Schultz-DuBois, Bell System Tech. J. $\underline{38}$, 271 (1959).

${ }^{15}$ A. M. Clogston, Phys. Rev. 118, 1229 (1960).

${ }^{16} \mathrm{~S}$. Geschwind, G. E. Devlin, R. L. Cohen, and S. R. Chinn, Phys. Rev. 137, A1087 (1965).

${ }^{17} \mathrm{~S}$. Geschwind, R. J. Collins, and A. L. Schawlow Phys. Rev. Letters $\underline{3}, 545$ (1959).

${ }^{18}$ I. D. Abella, A. Compaan, and L. Q. Lambert, Bull. Am. Phys. Soc. 14, 115 (1953).

${ }^{19}$ E. L. Hahn, Phys. Today 6,4 (1953).

${ }^{20}$ H. Y. Carr and E. M. Purcell, Phys. Rev. 94, 630 (1954).
} 